\title{
TENSOR PRODUCT OF MODULES OVER A VERTEX ALGEBRA
}

\author{
JOSÉ I. LIBERATI*
}

\begin{abstract}
We found a necessary and sufficient condition for the existence of the tensor product of modules over a vertex algebra. We defined the notion of vertex bilinear map and we provide two algebraic construction of the tensor product, where one of them is of ring theoretical type. We show the relation between the tensor product and the vertex homomorphisms. We prove the commutativity of the tensor product. We also prove the associativity of the tensor product of modules under certain necessary and sufficient condition. Finally, we show certain functorial properties of the vertex homomorphims and the tensor product.
\end{abstract}

\section{INTRODUCTION}

This is the first of a series of papers (the second one is [L1]) trying to extend certain restricted definitions and constructions developed for vertex operator algebras to the general framework of vertex algebras without assuming any grading condition neither on the vertex algebra nor on the modules involved, and making a strong emphasis on the commutative associative algebra point of view instead of the Lie theoretical point of view. These series of works help to eliminate the Jacobi identity from the theory of vertex algebras and clean it by considering the correct point of view based on the theory of commutative associative algebras.

The study of tensor product theory for representations of a chiral algebra (or vertex operator algebra), was initiated by physicists ([MS] and references there in). After that, Gaberdiel [G] pointed out that Borcherds suggested a ring like construction, and he developed it on a physical level of rigor. Simultaneously, Huang and Lepowsky [HL1]-[HL5] and [H], gave the first mathematically rigorous notion and construction of the tensor product of certain graded modules over certain vertex operator algebras, by using analytical methods. In [Li1], Li gave a formal variable approach to the tensor product theory, following classical Lie algebra theory and restricting the definitions and constructions to certain graded modules over a rational vertex operator algebra.

Many mathematicians believe that there should be an algebraic and ring type construction of the tensor product of modules over a vertex algebra. It has been a necessary and missing part of the theory in the last 30 years. The present work try to fill this gap.

In order to have a purely algebraic definition and construction, we loose the analytical and geometric interpretation, but we simplify the theory.

Our work is strongly influenced by the works of Haisheng Li, specially his thesis. Most of the ideas and tricks used in this work are taken from his papers, but applied in a different context. The ideas and constructions are very simples and naturals.

In section 2, we present the basic definitions and notations. In section 3 , we found a necessary and sufficient condition for the existence of the tensor product of modules over a vertex algebra, that we called the kernel intertwining operator full equality condition, and we present the first construction.

Since we have a different point of view of very well known notions, in section 4 we define the notion of vertex bilinear map that replaces the notion of intertwining operator. This allow us to

2010 Mathematics Subject Classification. Primary 17B69; Secondary 17B67.

*Ciem - CONICET, Medina Allende y Haya de la Torre, Ciudad Universitaria, (5000) Córdoba - Argentina.

e-mail: joseliberati@gmail.com. 
redefine the notion of tensor product with a ring theoretical interpretation (Definition 4.4), and we provide the second algebraic construction of the tensor product, which is of ring theoretical type.

In section 5, we introduce the notion of (right) vertex homomorphism, producing the "Hom" functor for modules over a vertex algebra, obtaining (from our point of view) an analog of the Hom functor for modules over an associative commutative algebra, and we denote it as Vhom. We prefer to think about it in this way, instead of the Hom functor in Lie theory. In the first part of this section, we follow [Li1], [Li2] and [DLM]. The most important functors in homological algebra are Hom, tensor product and functors derived from them. In the case of vertex algebras, we should consider Vhom (and later $V h o m^{r}$ ). We shall show that there is an intimate relationship between Vhom and tensor product, they form an adjoint pair of functors (see Theorem 5.6, Theorem 5.14 and Corollary 5.15).

In section 6 , we prove the commutativity of the tensor product. Then we prove the associativity of the tensor product under certain (algebraic and natural) necessary and sufficient condition. In order to prove the associativity, we shall use the universal property of the tensor product instead of the explicit construction, following in part the ideas in [DLM] but using algebraic methods instead of analytic ones. We simplify Huang's convergence assumptions.

In section 7 , we introduce the notions of vertex projective, vertex injective and vertex flat $V$-modules. Roughly speaking, homological algebra is concerned with the question of how much modules differ from being projective, injective and flat. We do not try to develop homological algebra theory for vertex algebras in this work. This is just an introduction or motivation to the reader to try to develop it from the point of view of a vertex algebra as a generalization of an associative commutative algebra with unit. The point of view and the results of this work could be the starting point of the development of homological algebra for vertex algebras.

The results and constructions of this work could be used to simplify the construction of the tensor product by Kazhdan and Lusztig in [KL1]-[KL5], by using the relation with the tensor product of modules over the vertex algebras associated to the affine Kac-Moody algebras founded by Zhang [Zha] and references there in. It is interesting to see possible consequences on the tensor product of modules over quantum groups.

In [L2], we translate the ideas of this work to the construction of the tensor product of conformal modules over a Lie conformal algebra, and in [L3] the construction is done for $\mathrm{H}$ pseudo algebras, answering an open question suggested by V. Kac during a graduate course at MIT in 1997.

After reading this work, there is no doubt that this is the correct point of view and that the notation that we introduced (many years ago) makes it an elegant work. On may say that many results of this paper follow by standard arguments and therefore it is not a valuable work, but the key part of this paper (and [L1]) is that we found the correct approach, and the consequence of this is that the main theorems follow by standard arguments.

Finally, for me it is important to point out that in 2011 we found the kernel condition and the first construction of the tensor product, but due to personal problems we abandoned this paper until January 2014, when we found the notion of vertex bilinear map and the second construction. We typed this work, including the commutativity property of the tensor product and the relation with Vhom. It was basically finished in 2014, except for the associativity and the final section. In May 2016, we resume this work by checking the typos, and we decided to complete it with the proof of associativity and the study of the functorial properties of Vhom and the tensor product written in the last section, producing this final version.

Unless otherwise specified, all vector spaces, linear maps and tensor products are considered over an algebraically closed field $\mathbf{k}$ of characteristic 0 . 


\section{Definitions AND NOTATION}

In order to make a self-contained paper, in this section we present the notion of vertex algebra and their modules, intertwining operators and tensor product. Our presentation and notation differ from the usual one because we want to emphasize the point of view that vertex algebras are analog to commutative associative algebras with unit.

Throughout this work, we define $(x+y)^{n}$ for $n \in \mathbb{Z}$ (in particular, for $n<0$ ) to be the formal series

$$
(x+y)^{n}=\sum_{k \in \mathbb{Z}_{+}}\left(\begin{array}{l}
n \\
k
\end{array}\right) x^{n-k} y^{k},
$$

where $\left(\begin{array}{l}n \\ k\end{array}\right)=\frac{n(n-1) \ldots(n-k+1)}{k !}$.

Definition 2.1. A vertex algebra is a quadruple $(V, \dot{z}, \mathbf{1}, d)$ that consists of a k-vector space $V$ equipped with a linear map

$$
\begin{aligned}
\dot{z}: V \otimes V & \longrightarrow V((z)) \\
a \otimes b & \longmapsto a_{\dot{z}} b,
\end{aligned}
$$

a distinguished vector 1 and $d \in \operatorname{End}(V)$ satisfying the following axioms $(a, b, c \in V)$ :

- Unit:

$$
\mathbf{1}_{\dot{z}} a=a \quad \text { and } \quad a_{\dot{z}} \mathbf{1}=e^{z d} a
$$

- Translation - Derivation:

$$
(d a)_{\dot{z}} b=\frac{d}{d z}\left(a_{\dot{z}} b\right), \quad d\left(a_{\dot{z}} b\right)=(d a)_{\dot{z}} b+a_{\dot{z}}(d b)
$$

- Commutativity:

$$
a_{\dot{z}} b=e^{z d}\left(b_{-\dot{z}} a\right)
$$

- Associativity: For any $a, b, c \in V$, there exists $l \in \mathbb{N}$ such that

$$
(z+w)^{l}\left(a_{\dot{z}} b\right)_{\dot{w}} c=(z+w)^{l} a_{z \dot{+} w}\left(b_{\dot{w}} c\right) .
$$

Remark 2.2. (a) Observe that the standard notation $Y(a, z) b$ for the $z$-product in (2.1) has been changed. We adopted this notation following the practical idea of the $\lambda$-bracket in the notion of Lie conformal algebra (also called vertex Lie algebra in the literature), see $[\mathrm{K}]$. In fact, we have been using this notation since 2011 (see the undergraduate thesis of my student in $[\mathrm{O}]$ ).

(b) If we write $a_{\dot{z}} b$ in terms of its coefficients we obtain the definition of the $n$-products $a_{n} b$ given by

$$
a_{\dot{z}} b=\sum_{n \in \mathbb{Z}}\left(a_{n} b\right) z^{-n-1} .
$$


(c) Sometimes we shall use the following standard notation for the vertex operators:

$$
a_{\dot{z}}=\sum_{n \in \mathbb{Z}} a_{n} z^{-n-1}
$$

where $a_{n} \in \operatorname{End}(V)$ is the endomorphism determined by the $n$-product. Since the image of the map $\dot{z}$ is in $V((z))$ we have that for all $a, b \in V$ there exists $N_{a, b} \in \mathbb{N}$ such that

$$
a_{\dot{z}} b=\sum_{n<N_{a, b}}\left(a_{n} b\right) z^{-n-1} \text {. }
$$

That is, for any $a, b \in V$, there exists $N_{a, b} \geq 0$ such that

$$
a_{n} b=0 \quad \forall n \geq N_{a, b} .
$$

(d) The associativity axiom can be replaced by the same identity (2.4), with the integer $l$ depending only on $a$ and $c$.

The commutativity axiom is known in the literature as skew-symmetry (see [LL, K]), but for us it really corresponds to commutativity. We want to emphasize the point of view of a vertex algebra as a generalization of an associative commutative algebra with unit (and a derivation), as in [BK, Li3, Bo2], and having in mind the usual (trivial) example of holomorphic vertex algebra given by any associative commutative algebra $A$ with unit $\mathbf{1}$ and a derivation $d$, with the $\dot{z}$-product defined by $a_{\dot{z}} b:=\left(e^{z d} a\right) \cdot b$. In the special case of $d=0$, we simply have an associative commutative algebra with unit and $a_{\dot{z}} b:=a \cdot b$, and with the definition presented before, it is immediate to see, without any computation, that this is a vertex algebra, and this is the reason for us to call the usual skew-symmetry axiom as commutativity.

An equivalent definition can be obtained by replacing the associativity axiom by the associator formula (which is equivalent to what is known in the literature as the iterate formula (see [LL], p.54-55) or the $n$-product identity (see $[\mathrm{BK}])$ ):

$$
\left(a_{\dot{z}} b\right)_{\dot{y}} c-a_{z \dot{+} y}\left(b_{\dot{y}} c\right)=b_{\dot{y}}\left(a_{y \dot{+} z} c-a_{z \dot{+} y} c\right) \quad \forall a, b, c \in V .
$$

Observe that in the last term of the associator formula we can not use linearity to write it as a difference of $b_{\dot{w}}\left(a_{w+z} c\right)$ and $b_{\dot{w}}\left(a_{z+\dot{w}} c\right)$, because neither of these expressions in general exist (see [LL], p.55). This alternative definition of vertex algebra using the (iterate formula or) the associator formula is essentially the original definition given by Borcherds [Bo1], but in our case it is written using the generating series in $z$ instead of the $n$-products.

Another equivalent definition can be obtained by replacing the associativity axiom (and commutativity) by the Jacobi identity:

$$
\delta(x-y, z) a_{\dot{x}} b_{\dot{y}}-\delta(-y+x, z) b_{\dot{y}} a_{\dot{x}}=\delta(x-z, y)\left(a_{\dot{z}} b\right)_{\dot{y}} \quad \forall a, b \in V,
$$

where $\delta(x, y):=\sum_{n \in \mathbb{Z}} x^{n} y^{-n-1}$ is the delta function.

It is well known that taking $\operatorname{Res}_{x}$ (that is the coefficient in $x^{-1}$ ) of the Jacobi identity we obtain the Associator formula and, using Remark 2.2 (c), by multiplying the Associator formula (2.5) by $(y+z)^{N_{a, c}}$ we get Associativity. The rest of the proof of the equivalence between these definitions is more complicated (see [LL]). It is well known (see [LL]) that in the three equivalent definitions that we presented, some of the axioms can be obtained from the others, but we prefer to make emphasis on the properties of $d$ and the explicit formula for the multiplication by the unit.

Another useful formula, called the Commutator formula, is obtained by taking $\operatorname{Res}_{z}$ in the Jacobi identity:

$$
a_{\dot{x}}\left(b_{\dot{y}} c\right)-b_{\dot{y}}\left(a_{\dot{x}} c\right)=\left(a_{x-y} b-a_{-\dot{y}+x} b\right)_{\dot{y}} c .
$$


from which it is easy to deduce what is called weak commutativity or locality in the literature: for all $a, b \in V$, there exists $k \in \mathbb{N}$ such that

$$
(x-y)^{k} a_{\dot{x}}\left(b_{\dot{y}} c\right)=(x-y)^{k} b_{\dot{y}}\left(a_{\dot{x}} c\right) \quad \text { for all } c \in V .
$$

Definition 2.3. A module over a vertex algebra $V$ is a k-vector space $M$ equipped with an endomorphism $d$ of $M$ and a linear map

$$
V \otimes M \longrightarrow M((z)), \quad(a, u) \longmapsto a_{\dot{z}}^{M} u=\sum_{n \in \mathbb{Z}}\left(a_{n} u\right) z^{-n-1}
$$

satisfying the following axioms $(a, b \in V$ and $u \in M)$ :

- Unit:

$$
\mathbf{1}_{\dot{z}}^{M} u=u
$$

- Translation - Derivation:

$$
(d a)_{\dot{z}}^{M} u=\frac{d}{d z}\left(a_{\dot{z}}^{M} u\right), \quad d\left(a_{\dot{z}}^{M} u\right)=(d a)_{\dot{z}}^{M} u+a_{\dot{z}}^{M}(d u)
$$

- Associativity: For any $a, b \in V$ and $u \in M$, there exists $l \in \mathbb{N}$ such that

$$
(z+x)^{l}\left(a_{\dot{z}} b\right)_{\dot{x}}^{M} u=(z+x)^{l} a_{z \dot{+} x}^{M}\left(b_{\dot{x}}^{M} u\right) .
$$

Remark 2.4. (a) Sometimes, if everything is clear, we shall use $a_{\dot{z}} u$ instead of $a_{\dot{z}}^{M} u$. We shall also use the notation $a_{\dot{z}}^{M}$. Obviously, $V$ is a module over $V$.

(b) We follow [L1, BK], in the definition of module, because in order to talk about intertwining operators we need to work with this category of modules, namely, with this $\mathbf{k}[d]$-module structure (similar to the situation of Lie conformal algebras $[\mathrm{K}]$ ). We denote it by $d$, but it should be $d_{M}$. (c) Similarly to the definition of vertex algebra, the associativity axiom could be replaced by the associator formula:

$$
\begin{aligned}
\left(a_{\dot{z}} b\right)_{\dot{y}} v & =a_{z \dot{+} y}\left(b_{\dot{y}} v\right)+b_{\dot{y}}\left(a_{y \dot{+} z} v-a_{z \dot{+} y} v\right) \\
& =a_{z \dot{+} y}\left(b_{\dot{y}} v\right)-\operatorname{Res}_{x} \delta(-y+x, z) b_{\dot{y}}\left(a_{\dot{x}} v\right) .
\end{aligned}
$$

or by the Jacobi identity. As in the vertex algebra case, the associativity axiom can be replaced by the same identity (2.10), with the integer $l$ depending only on $a$ and $u$. Any $V$-module $M$ satisfies the Commutator formula for modules given by (for any $a, b \in V$ and $u \in M$ ):

$$
a_{\dot{x}}\left(b_{\dot{y}} u\right)-b_{\dot{y}}\left(a_{\dot{x}} u\right)=\left(a_{x-y} b-a_{-\dot{y}+x} b\right)_{\dot{y}} u \text {. }
$$

from which it is easy to deduce what is called weak commutativity or locality for a $V$-module $M$ : for all $a, b \in V$, there exists $k \in \mathbb{N}$ such that

$$
(x-y)^{k} a_{\dot{x}}\left(b_{\dot{y}} u\right)=(x-y)^{k} b_{\dot{y}}\left(a_{\dot{x}} u\right) \quad \text { for all } u \in M .
$$

It is well known that commutator formula for modules can not be used to replace associativity axiom (commutativity of left multiplication operators does not imply module structure over an associative commutative algebra).

(d) For $a \in V$ and $u \in M$, we take $N_{a, u}$ the minimum positive integer such that

$$
z^{N_{a, u}}\left(a_{\dot{z}}^{M} u\right) \in M[[z]] .
$$


(e) If $V$ is an associative commutative algebra with unit and $d=0$, then (by translation axiom) we have that $\frac{d}{d z}\left(a_{\dot{z}}^{M} u\right)=0$. Hence $a_{\dot{z}}^{M} u$ is independent of $z$ and a module over the vertex algebra $V$ corresponds to a module over the associative commutative algebra with unit $V$ together with $d=d_{M} \in \operatorname{End}_{V}(M)$. If $d_{M}=0$, then we recover the usual notion of module over an associative commutative algebra with unit.

Let $M$ and $N$ be $V$-modules, a $V$-homomorphism or a homomorphism of $V$-modules from $M$ to $N$ is a linear map $\varphi: M \rightarrow N$ such that for $a \in V$ and $u \in M$

$$
\varphi\left(a_{\dot{z}} u\right)=a_{\dot{z}} \varphi(u) \text { and } \varphi(d u)=d \varphi(u) \text {. }
$$

The space of homomorphisms of $V$-modules is denoted $\operatorname{Hom}_{V}(M, N)$.

We shall need the following well known results:

Proposition 2.5. (a) Let $V$ be a vertex algebra. Then the quotient space

$$
\mathfrak{g}(V):=\mathbf{k}\left[t, t^{-1}\right] \otimes V /\left(\frac{d}{d t} \otimes 1+1 \otimes d\right)\left(\mathbf{k}\left[t, t^{-1}\right] \otimes V\right),
$$

is a Lie algebra associated to $V$ with Lie bracket:

$$
\left[\overline{t^{m} \otimes a}, \overline{t^{n} \otimes b}\right]=\sum_{i=0}^{\infty}\left(\begin{array}{c}
m \\
i
\end{array}\right) \overline{t^{m+n-i} \otimes a_{i} b} .
$$

for any $a, b \in V$.

(b) Any $V$-module is a $\mathfrak{g}(V)$-module, where $t^{m} \otimes a$ acts as $a_{m}$.

(c) For any $V$-module $M$, define $\widehat{M}=\mathbf{k}\left[t, t^{-1}\right] \otimes M$. Then $\widehat{M}$ is a $\mathfrak{g}(V)$-module with the action given by:

$$
\left(t^{m} \otimes a\right)\left(t^{n} \otimes v\right)=\sum_{i=0}^{\infty}\left(\begin{array}{c}
m \\
i
\end{array}\right) t^{m+n-i} \otimes a_{i} v
$$

for any $a \in V$ and $v \in M$.

As an abuse of notation, we remove the bar in the class elements in $\mathfrak{g}(V)$.

Now, we introduce the notions of intertwining operators and tensor product of modules, and both notions will be redefined from a ring theoretical point of view in Section 4 .

Definition 2.6. Let $M, N$ and $W$ be three $V$-modules. An intertwining operator of type $\left({ }_{M, N}^{W}\right)$ is a $\mathbf{k}$-bilinear map

$$
\begin{aligned}
I_{z}: M \times N & \longrightarrow W((z)) \\
(u, v) & \longmapsto I_{z}(u, v)=\sum_{n \in \mathbb{Z}} I_{(n)}(u, v) z^{-n-1},
\end{aligned}
$$

satisfying the following conditions:

- Translation - Derivation:

$$
I_{z}(d u, v)=\frac{d}{d z} I_{z}(u, v), \quad \text { and } d\left(I_{z}(u, v)\right)=I_{z}(d u, v)+I_{z}(u, d v),
$$

- Jacobi identity: For all $a \in V, u \in M$ and $v \in N$

$$
\delta(x-y, z) a_{\dot{x}} I_{y}(u, v)-\delta(-y+x, z) I_{y}\left(u, a_{\dot{x}} v\right)=\delta(x-z, y) I_{y}\left(a_{\dot{z}} u, v\right)
$$


Definition 2.7. Let $M$ and $N$ be two $V$-modules. A pair $\left(M \underset{V}{\otimes} N, F_{z}\right)$, which consists of a $V$ - module $M \underset{V}{\otimes} N$ and an intertwining operator $F_{z}$ of type $\left(\begin{array}{c}M \otimes N \\ M, N\end{array}\right)$, is called a tensor product for the ordered pair $(M, N)$ if the following universal property holds: For any $V$-module $W$ and any intertwining operator $I_{z}$ of type $\left(\begin{array}{c}W \\ M, N\end{array}\right)$ there exists a unique $V$-homomorphism $\varphi$ from $M \otimes N$ to $W$ such that $I_{z}=\varphi \circ F_{z}$, where $\varphi$ is extended canonically to a linear map from $(M \underset{V}{\otimes} N)((z))$ to $W((z))$.

Just as in the classical algebra theory, it follows from the universal property that if there exists a tensor product for the ordered pair $(M, N)$, then it is unique up to a $V$-module isomorphism. Namely, the pair $\left(W, G_{z}\right)$ is another tensor product if and only if there exists a $V$-module isomorphism $\phi: M \underset{V}{\otimes} N \rightarrow W$ such that $G_{z}=\phi \circ F_{z}$.

Using the Jacobi identity (2.16), it is immediate that any intertwining operator $I_{z} \in\left(\begin{array}{c}W \\ M, N\end{array}\right)$ satisfies the associator formula (for $a \in V, u \in M$ and $v \in N$ )

$$
a_{z \dot{+} x} I_{x}(u, v)=I_{x}\left(a_{\dot{z}} u, v\right)+I_{x}\left(u, a_{z \dot{+} x} v-a_{x \dot{+} z} v\right),
$$

the iterated formula

$$
I_{z}\left(a_{\dot{x}} u, v\right)=\operatorname{Res}_{y}\left[\delta(y-z, x) a_{\dot{y}} I_{z}(u, v)-\delta(-z+y, x) I_{z}\left(u, a_{\dot{y}} v\right)\right],
$$

and the commutator formula

$$
a_{\dot{x}} I_{y}(u, v)-I_{y}\left(u, a_{\dot{x}} v\right)=I_{y}\left(a_{x-y} u-a_{-\dot{y}+x} u, v\right) .
$$

\section{First construction of the tensor product}

First of all, we need some definitions in order to find necessary and sufficient conditions for the existence of the tensor product.

Definition 3.1. (a) Let $M$ and $N$ be two $V$-modules. We define the kernel of the intertwining operators from the pair $(M, N)$ as follows

$$
\begin{aligned}
\operatorname{Ker}\left(\begin{array}{c}
\cdot \\
M, N
\end{array}\right):=\{ & (u, v) \in M \times N \mid \exists l_{u, v} \in \mathbb{N} \text { such that } I_{(n)}(u, v)=0 \\
& \text { for all } \left.n \geq l_{u, v}, \text { for all } V \text {-modules } W \text {, and for all } I_{z} \in\left(\begin{array}{c}
W \\
M, N
\end{array}\right)\right\}
\end{aligned}
$$

(b) We say that the pair $(M, N)$ satisfies the kernel intertwining operator full equality condition if

$$
M \times N=\operatorname{Ker}\left(\begin{array}{c}
\cdot \\
M, N
\end{array}\right) .
$$

Proposition 3.2. (Necessary condition) If the tensor product $\left(M \underset{V}{\otimes} N, F_{z}\right)$ exists, then the pair $(M, N)$ satisfies the kernel intertwining operator full equality condition. 
Proof. Fix $(u, v) \in M \times N$ and using that $F_{z}(u, v) \in(M \underset{V}{\otimes} N)((z))$, we have that there exists $N \in \mathbb{N}$ (depending on $u$ and $v$ ) such that $F_{(n)}(u, v)=0$ for all $n \geq N$. Since for any $V$-module $W$ and any intertwining operator $I_{z}$ of type $\left(\begin{array}{c}W \\ M, N\end{array}\right)$ there exists a unique $V$-homomorphism $\varphi$ from $M \underset{V}{\otimes} N$ to $W$ such that $I_{z}=\varphi \circ F_{z}$, where $\varphi$ is extended canonically to a linear map from $(M \underset{V}{\otimes} N)^{V}((z))$ to $W((z))$, it follows that $I_{(n)}(u, v)=0$ for all $n \geq N$.

Theorem 3.3. (Sufficient condition) If the pair $(M, N)$ satisfies the kernel intertwining operator full equality condition, then the tensor product $\left(M \underset{V}{\otimes} N, F_{z}\right)$ exists.

We shall do the construction of the tensor product in the rest of this section, and we shall see that it is also a sufficient condition for the existence of the tensor product.

Lemma 3.4. (Lemma 5.1.3, [Li1]) Let $\left(M \underset{V}{\otimes} N, F_{z}\right)$ be a tensor product of the ordered pair $(M, N)$. Then $F_{z}$ is surjective in the sense that all the coefficients of $F_{z}(u, v)$, for $u \in M$ and $v \in N$, linearly span $M \underset{V}{\underset{N}{\otimes}} N$.

With this lemma in mind, the essential idea is to consider "strings over $\mathbb{Z}$ " for each pair $(u, v) \in M \times N$ and then take the quotient by all the necessary conditions in order to get an intertwining operator by taking the generating series of these strings (cf. [Li1]).

Therefore, let $M$ and $N$ be two $V$-modules, and set

$$
F_{0}(M, N)=\mathbb{C}\left[t, t^{-1}\right] \otimes M \otimes N .
$$

As usual in vertex algebra theory, it is more clear to work with generating series in order to manipulate string of vectors. For this reason we introduce the following very important notation for any $u \in M$ and $v \in N$ (cf. formula (5.2.2) in [Li1]):

$$
u \otimes_{z} v:=\delta(z, t) \otimes u \otimes v=\sum_{n \in \mathbb{Z}}\left(t^{n} \otimes u \otimes v\right) z^{-n-1} .
$$

We want to take the necessary quotients in order to obtain that $\underset{z}{\otimes}$ is an intertwining operator. In particular, it should satisfies the commutator formula (2.19), and this is the motivation for the following action, but in fact, we will see that it is not a Lie theoretical type action.

By using the generating series of elements in $\mathfrak{g}(V)$ given by

$$
a_{\dot{z}}=\delta(z, t) \otimes a=\sum_{n \in \mathbb{Z}}\left(t^{n} \otimes a\right) z^{-n-1},
$$

we define an action of $\mathfrak{g}(V)$ on $F_{0}(M, N)$ as follows (for $a \in V, u \in M, v \in N$ ):

$$
\begin{aligned}
a_{\dot{z}}\left(u \otimes_{y} v\right) & =u \underset{y}{\otimes}\left(a_{\dot{z}} v\right)+\left(a_{z-y} u-a_{-y+z} u\right) \underset{y}{\otimes} v \\
& =u \otimes_{y}\left(a_{\dot{z}} v\right)+\operatorname{Res}_{x} \delta(z-x, y)\left(a_{\dot{x}} u \underset{y}{\otimes} v\right)
\end{aligned}
$$

(cf. formulas of $\Delta_{z}$ in p. 183 [MS] and (5.2.6) in [Li1]), and define the linearly extended map given on generators by $d(u \underset{z}{\otimes} v)=d u \underset{z}{\otimes} v+u \underset{z}{\otimes} d v$.

Proposition 3.5. Under the above defined action, $F_{0}(M, N)$ is a $\mathfrak{g}(V)$-module satisfying

$$
\mathbf{1}_{\dot{z}}=\mathrm{id}, \quad(d a)_{\dot{z}}=\frac{d}{d z} a_{\dot{z}}, \quad \text { and }\left[d, a_{\dot{z}}\right]=(d a)_{\dot{z}}
$$

Moreover, $F_{0}(M, N)$ is just the tensor product of Lie algebra modules $\widehat{M}$ and $N$ (cf. Proposition 2.5). 
Proof. Expanding the coefficients in (3.2), we obtain:

$$
\left(t^{m} \otimes a\right)\left(t^{n} \otimes u \otimes v\right)=t^{n} \otimes u \otimes a_{m} v+\sum_{i=0}^{\infty}\left(\begin{array}{c}
m \\
i
\end{array}\right) t^{m+n-i} \otimes a_{i} u \otimes v .
$$

Using Proposition 2.5, we have the $\mathfrak{g}(V)$-module structure given by the tensor product of Lie algebra modules. The other properties follow by straightforward computations.

Let $J_{0}$ be the $\mathfrak{g}(V)$-submodule of $F_{0}(M, N)$ generated by the following subspace:

$\mathbf{k}-\operatorname{span}\left\{t^{n} \otimes u \otimes v \in F_{0}(M, N) \mid I_{(n)}(u, v)=0\right.$ for all $V$-modules $W$, and for all $\left.I_{z} \in\left(\begin{array}{c}W \\ M, N\end{array}\right)\right\}$

Since $M \times N=\operatorname{Ker}(\underset{M, N}{\dot{*}})$, then for every $(u, v) \in M \times N$, there exists $l \in \mathbb{N}$ such that $I_{(n)}(u, v)=0$ for all $n \geq l$, for all $V$-modules $W$, and for all $I_{z} \in\left(\begin{array}{c}W \\ M, N\end{array}\right)$.

Now, we take

$$
F_{1}(M, N)=F_{0}(M, N) / J_{0}
$$

We will still use the notation $u \otimes \otimes_{x} v$ and $t^{n} \otimes u \otimes v$ for elements in the quotient space $F_{1}(M, N)\left[\left[x, x^{-1}\right]\right]$ and $F_{1}(M, N)$ respectively. We have the following important result:

Proposition 3.6. For any $a \in V$ and any $t^{n} \otimes u \otimes v \in F_{1}(M, N)$, we have

(a) $a_{\dot{z}}\left(t^{n} \otimes u \otimes v\right)$ involves only finitely many negative powers of $z$.

(b) $u \otimes_{x} v$ involves only finitely many negative powers of $x$.

(c) Let $a \in V, u \in M$ and $v \in N$. Then for any integer $k$ such that $k \geq N_{a, u}$ (see (2.14)), we have

$$
(z-x)^{k} a_{\dot{z}}\left(u \otimes_{x} v\right)=(z-x)^{k}\left(u \underset{x}{\otimes} a_{\dot{z}} v\right) \in F_{1}(M, N)((z, x)) .
$$

(d) For any integer $k$ such that $k \geq N_{a, u}$, an alternative definition for the action of $\mathfrak{g}(V)$ (or $V)$ in $F_{1}(M, N)$ is given by

$$
a_{\dot{z}}(u \underset{x}{\otimes} v)=(z-x)^{-k}\left((z-x)^{k}\left(u \underset{x}{\otimes} a_{\dot{z}} v\right)\right) .
$$

similar to the ring theoretical case.

(e) The map $d$ is well defined in $F_{1}(M, N)$, that is $d\left(J_{0}\right) \subseteq J_{0}$.

Proof. (a) We fix $a \in V$ and $t^{n} \otimes u \otimes v \in F_{1}(M, N)$. Recall formula (3.3):

$$
\left(t^{m} \otimes a\right)\left(t^{n} \otimes u \otimes v\right)=t^{n} \otimes u \otimes a_{m} v+\sum_{i=0}^{\infty}\left(\begin{array}{c}
m \\
i
\end{array}\right) t^{m+n-i} \otimes a_{i} u \otimes v .
$$

Observe that in the first term, $a_{m} v=0$ for a sufficiently large $m$, and in the second term, the sum is finite and independent of $m$. Then for each element $a_{i} u \otimes v$ there exists a power of $t$ such that $t^{l} \otimes a_{i} u \otimes v=0$ if $l$ is large enough. Therefore, for a large enough $m$ the result is proved.

(b) It is immediate from the definition of $J_{0}$ and the kernel condition that is assume for the pair $(M, N)$.

(c) Multiplying (3.2) by $(z-x)^{N_{a, u}}$, with $N_{a, u}$ as in (2.14), we obtain the equality. Since the LHS is an element in $\left(F_{1}(M, N)\right)((z))((x))$ and the RHS is in $\left(F_{1}(M, N)\right)((x))((z))$, we get the result. 
(d) Note that the LHS in (c) involve only finitely many negative power of $x$, then we multiply both sides by $(z-x)^{-k}$. On the other hand, (c) implies that $(z-x)^{k}\left(u \otimes_{x} a_{\dot{z}} v\right) \in$ $F_{1}(M, N)((z, x))$, so that the expression on the RHS of $(\mathrm{d})$ is well defined. Now, it remains to prove that it is independent of $k$. Assume that $k_{1} \geq k_{2} \geq N_{a, u}$. Then for $i=1,2$, we have $(z-x)^{k_{i}}\left(u \otimes_{x} a_{\dot{z}} v\right) \in F_{1}(M, N)((z, x))$ and

$$
\begin{aligned}
(z-x)^{-k_{1}}\left((z-x)^{k_{1}}\left(u \otimes_{x} a_{\dot{z}} v\right)\right) & =(z-x)^{-k_{1}}\left((z-x)^{k_{1}-k_{2}}\left((z-x)^{k_{2}}\left(u \otimes_{x} a_{\dot{z}} v\right)\right)\right) \\
& =(z-x)^{-k_{1}}(z-x)^{k_{1}-k_{2}}\left((z-x)^{k_{2}}\left(u{\underset{x}{x}}_{\dot{z}} a\right)\right) \\
& =(z-x)^{-k_{2}}\left((z-x)^{k_{2}}\left(u \otimes_{x} a_{\dot{z}} v\right)\right),
\end{aligned}
$$

proving the assertion (cf. similar arguments to those in Remark 5.17 and the proof of Lemma 5.10 in $[\mathrm{Li3}])$.

(e) Suppose $t^{n} \otimes u \otimes v \in J_{0}$, then $d\left(t^{n} \otimes u \otimes v\right)=t^{n} \otimes d u \otimes v+t^{n} \otimes u \otimes d v$. Using (2.15), we have $I_{(n)}((d u, v)+(u, d v))=d I_{(n)}(u, v)=0$, finishing the proof.

Now, let $J_{1}$ be the $\mathfrak{g}(V)$-submodule of $F_{1}(M, N)$ linearly spanned by all the coefficients in the following expressions:

$$
\begin{gathered}
\delta(x-y, z) a_{\dot{x}}\left(u \otimes_{y} v\right)-\delta(-y+x, z)\left(u \otimes_{y} a_{\dot{x}} v\right)-\delta(x-z, y)\left(a_{\dot{z}} u \otimes_{y} v\right), \\
\left(d u \otimes_{z} v\right)-\frac{d}{d z}(u \underset{z}{\otimes} v) .
\end{gathered}
$$

for $a \in V, u \in M, v \in N$. By straightforward computations, it is easy to see that $J_{1}$ is invariant by $d$. We define

$$
M \underset{V}{\otimes} N=F_{1}(M, N) / J_{1}
$$

Proposition 3.7. The space $M \underset{V}{\otimes} N$ is a $V$-module, and $\underset{z}{\otimes}$ is an intertwining operator of type $\left(\begin{array}{c}M \otimes N \\ M, N\end{array}\right)$.

Proof. By Proposition 3.6, and the $J_{1}$-defining relations, it is clear that $\otimes$ is an intertwining operator. In order to see that $M \otimes_{V} N$ is a $V$-module, by Proposition 3.5 , it remains to check Jacobi identity following the proof of Theorem 5.2.9 in [Li1]. For $a, b \in V, u \in M, v \in N$, we have:

$$
\begin{aligned}
& \delta(z-w, x) a_{\dot{z}}\left(b_{\dot{w}}\left(u \otimes_{y} v\right)\right)=\delta(z-w, x) a_{\dot{z}}\left(u \otimes_{y} b_{\dot{w}} v+\operatorname{Res}_{r} \delta(w-r, y)\left(b_{\dot{r}} u \otimes_{y} v\right)\right) \\
& =\delta(z-w, x)\left[u \otimes_{y} a_{\dot{z}}\left(b_{\dot{w}} v\right)+\operatorname{Res}_{r} \delta(z-r, y)\left(a_{\dot{r}} u \underset{y}{\otimes_{y}} b_{\dot{w}} v\right)+\operatorname{Res}_{r} \delta(w-r, y) a_{\dot{z}}\left(b_{\dot{r}} u \otimes_{y} v\right)\right]
\end{aligned}
$$

and

$$
\begin{aligned}
& \delta(-w+z, x) b_{\dot{w}}\left(a_{\dot{z}}\left(u \otimes_{y} v\right)\right)=\delta(-w+z, x) b_{\dot{w}}\left(u \otimes_{y} a_{\dot{z}} v+\operatorname{Res}_{r} \delta(z-r, y)\left(a_{\dot{r}} u \otimes_{y} v\right)\right) \\
& =\delta(-w+z, x)\left[u \otimes_{y} b_{\dot{w}}\left(a_{\dot{z}} v\right)+\operatorname{Res}_{r} \delta(w-r, y)\left(b_{\dot{r}} u \otimes_{y} a_{\dot{z}} v\right)+\operatorname{Res}_{r} \delta(z-r, y) b_{\dot{w}}\left(a_{\dot{r}} u \otimes_{y} v\right)\right]
\end{aligned}
$$

and, on the other hand

$$
\delta(z-x, w)\left(a_{\dot{x}} b\right) \dot{w}\left(u \otimes_{y} v\right)=\delta(z-x, w)\left[u \otimes_{y}\left(a_{\dot{x}} b\right)_{\dot{w}} v+\operatorname{Res}_{r} \delta(w-r, y)\left(\left(a_{\dot{x}} b\right)_{\dot{r}} u \otimes_{y} v\right)\right]
$$


It follows from the Jacobi identity of $N$ that $\left(1^{\text {st }}\right.$ term in $\left.(3.4)\right)-\left(1^{\text {st }}\right.$ term in $\left.(3.5)\right)=\left(1^{\text {st }}\right.$ term in (3.6)). Using that

$$
\begin{aligned}
\delta(z-w, x) \delta(w-r, y) & =\delta(z-(y+r), x) \delta(w-r, y)=\delta(z-y, x+r) \delta(w-r, y) \\
\delta(-w+z, x) \delta(w-r, y) & =\delta(-(y+r)+z, x) \delta(w-r, y)=\delta(-y+z, x+r) \delta(w-r, y)
\end{aligned}
$$

and the defining relations of $J_{1}$, we obtain that $\left(3^{r d}\right.$ term in (3.4) $)-\left(2^{\text {nd }}\right.$ term in (3.5)) is equal to:

$$
\begin{aligned}
\operatorname{Res}_{r} & \delta(w-r, y)\left[\delta(z-y, x+r) a_{\dot{z}}\left(b_{\dot{r}} u \underset{y}{\otimes} v\right)-\delta(-y+z, x+r)\left(b_{\dot{r}} u \underset{y}{\otimes} a_{\dot{z}} v\right)\right]= \\
& =\operatorname{Res}_{r} \delta(w-r, y) \delta(z-(x+r), y)\left(a_{x \dot{r}}\left(b_{\dot{r}} u\right) \underset{y}{\otimes} v\right) \\
& =\operatorname{Res}_{r} \operatorname{Res}_{s} \delta(w-r, y) \delta(z-(x+r), y) \delta(x+r, s)\left(a_{\dot{s}}\left(b_{\dot{r}} u\right) \underset{y}{\otimes} v\right) \\
& =\operatorname{Res}_{r} \operatorname{Res}_{s} \delta(w-r, y) \delta(z-s, y) \delta(s-r, x)\left(a_{\dot{s}}\left(b_{\dot{r}} u\right) \underset{y}{\otimes} v\right) .
\end{aligned}
$$

Similarly, we have that $\left(2^{\text {nd }}\right.$ term in $\left.(3.4)\right)-\left(3^{\text {rd }}\right.$ term in $\left.(3.5)\right)$ is equal to:

$$
\begin{aligned}
\operatorname{Res}_{r} & \delta(z-r, y)\left[-\delta(-y+w,-x+r)\left(a_{\dot{r}} u \underset{y}{\otimes_{y}} b_{\dot{w}} v\right)+\delta(w-y,-x+r) b_{\dot{w}}\left(a_{\dot{r}} u \underset{y}{\otimes} v\right)\right]= \\
& =\operatorname{Res}_{r} \delta(z-r, y) \delta(w+(x-r), y)\left(b_{-x+r}\left(a_{\dot{r}} u\right) \underset{y}{\otimes} v\right) \\
& =\operatorname{Res}_{r} \operatorname{Res}_{s} \delta(z-r, y) \delta(w-s, y) \delta(-x+r, s)\left(b_{\dot{s}}\left(a_{\dot{r}} u\right) \underset{y}{\otimes} v\right) \\
& =-\operatorname{Res}_{r} \operatorname{Res}_{s} \delta(z-s, y) \delta(w-r, y) \delta(-r+s, x)\left(b_{\dot{r}}\left(a_{\dot{s}} u\right) \underset{y}{\otimes} v\right) .
\end{aligned}
$$

Therefore, using Jacobi identity of $M$, we have that $\left(3^{\text {rd }}\right.$ term in (3.4) $-2^{\text {nd }}$ term in $(3.5)+2^{\text {nd }}$ term in (3.4) $-3^{\text {rd }}$ term in (3.5)) is equal to

$$
\begin{aligned}
& \operatorname{Res}_{r} \operatorname{Res}_{s} \delta(z-s, y) \delta(w-r, y) \delta(s-x, r)\left(\left(a_{\dot{x}} b\right)_{\dot{r}} u \underset{y}{\otimes} v\right)= \\
& =\operatorname{Res}_{r} \delta(z-(r+x), y) \delta(w-r, y)\left(\left(a_{\dot{x}} b\right)_{\dot{r}} u \underset{y}{\otimes} v\right) \\
& =\operatorname{Res}_{r} \delta(y+(r+x), z) \delta(w-r, y)\left(\left(a_{\dot{x}} b\right)_{\dot{r}} u \underset{y}{\otimes} v\right) \\
& =\operatorname{Res}_{r} \delta(w+x, z) \delta(w-r, y)\left(\left(a_{\dot{x}} b\right)_{\dot{r}} u \underset{y}{\otimes} v\right) \\
& =\operatorname{Res}_{r} \delta(z-x, w) \delta(w-r, y)\left(\left(a_{\dot{x}} b\right)_{\dot{r}} u \underset{y}{\otimes} v\right)
\end{aligned}
$$

which is the same as the second term in (3.6), finishing the proof.

Now, it is clear from the construction that we have

Theorem 3.8. If the pair $(M, N)$ satisfies the kernel intertwining operator full equality condition, then the pair $(M \underset{V}{\otimes} N, \underset{z}{\otimes})$ is a tensor product of the pair $(M, N)$.

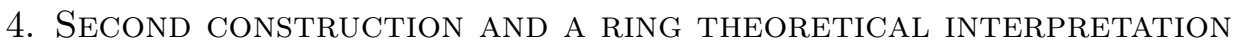

One of the motivations for the definition of vertex bilinear map is the following: let $R$ be a commutative (associative) ring, then the product $\mu$ in $R$ is an $R$-bilinear map, that is

$$
r \mu(a, b)=\mu(r a, b)=\mu(a, r b) \quad \text { for } \quad r, a, b \in R,
$$

which means that

$$
r(a b)=(r a) b \quad \text { and } \quad r(a b)=a(r b),
$$


observe that the first one corresponds to associativity (in our case (4.1)), and the second one is the commutativity of left multiplication operators, that in our case corresponds to locality, that is $(4.2)$.

By standard arguments in vertex algebra theory, we have that the following three definitions are equivalent to each other and they are equivalent to the notion of intertwining operator (see Theorem 4.2 below).

Definition 4.1. (1) Let $M, N$ and $W$ be three $V$-modules. A k-bilinear map $F_{z}: M \times N \rightarrow$ $W((z))$ is called a vertex bilinear map or $V$ - bilinear of type $(M, N ; W)$ if it satisfies:

(a) $F_{z}(d u, v)=\frac{d}{d z} F_{z}(u, v)$ and $F_{z}(u, d v)=\left(d-\frac{d}{d z}\right) F_{z}(u, v)$.

(b) For any $a \in V, u \in M$ and $v \in N$, there exist $n, k \in \mathbb{N}$ such that

$$
(z+x)^{n} a_{z \dot{+} x} F_{x}(u, v)=(z+x)^{n} F_{x}\left(a_{\dot{z}} u, v\right)
$$

and

$$
(z-x)^{k} a_{\dot{z}} F_{x}(u, v)=(z-x)^{k} F_{x}\left(u, a_{\dot{z}} v\right) .
$$

(2) Let $M, N$ and $W$ be three $V$-modules. A k-bilinear map $F_{z}: M \times N \rightarrow W((z))$ is called a vertex bilinear map or $V$-bilinear of type $(M, N ; W)$ if it satisfies:

(a) $F_{z}(d u, v)=\frac{d}{d z} F_{z}(u, v)$ and $F_{z}(u, d v)=\left(d-\frac{d}{d z}\right) F_{z}(u, v)$.

(b) For any $a \in V, u \in M$ and $v \in N$

$$
(z+x)^{N_{a, v}} a_{z \dot{+} x} F_{x}(u, v)=(z+x)^{N_{a, v}} F_{x}\left(a_{\dot{z}} u, v\right)
$$

and

$$
(z-x)^{N_{a, u}} a_{\dot{z}} F_{x}(u, v)=(z-x)^{N_{a, u}} F_{x}\left(u, a_{\dot{z}} v\right) .
$$

(3) Let $M, N$ and $W$ be three $V$-modules. A k-bilinear map $F_{z}: M \times N \rightarrow W((z))$ is called a vertex bilinear map or $V$-bilinear of type $(M, N ; W)$ if it satisfies:

(a) $F_{z}(d u, v)=\frac{d}{d z} F_{z}(u, v)$ and $F_{z}(u, d v)=\left(d-\frac{d}{d z}\right) F_{z}(u, v)$.

(b) For any $a \in V, u \in M$ and $v \in N$, there exist $n \in \mathbb{N}$ depending on $a$ and $v$, and $k \in \mathbb{N}$ depending on $a$ and $u$ such that

$$
(z+x)^{n} a_{z \dot{+} x} F_{x}(u, v)=(z+x)^{n} F_{x}\left(a_{\dot{z}} u, v\right)
$$

and

$$
(z-x)^{k} a_{\dot{z}} F_{x}(u, v)=(z-x)^{k} F_{x}\left(u, a_{\dot{z}} v\right) .
$$

We denote by $V$-Bilinear $(M, N ; W)$ the space of all vertex bilinear maps of this type. The other motivation for the definition of vertex bilinear map is the following result:

Theorem 4.2. The three definitions of vertex bilinear map are equivalent to each other. And, an intertwining operator of type $\left(\begin{array}{c}W \\ M, N\end{array}\right)$ is the same as a vertex bilinear map of type $(M, N ; W)$. 
Proof. It is clear that the Jacobi identity (2.16) imply (4.3) and (4.4) in the second definition of vertex bilinear map, by using the associator formula (2.17) and the commutator formula (2.19). It is also clear that the second definition imply the third, and the third imply the first one. So, it remains to show that (4.1) and (4.2) imply the Jacobi identity (2.16). But, this is immediate by using the following lemma (cf. Proposition 3.4.3 in [LL]):

Lemma 4.3. (Lemma 2.1, [Li3]) Let $U$ be a vector space and let

$$
\begin{aligned}
& A(x, y) \in U((x))((y)) \\
& B(x, y) \in U((y))((x)) \\
& C(z, y) \in U((y))((z)) .
\end{aligned}
$$

Then

$$
\delta(x-y, z) A(x, y)-\delta(-y+x, z) B(x, y)=\delta(x-z, y) C(z, y)
$$

if and only if there exist $k, l \in \mathbb{N}$ such that

$$
\begin{aligned}
(x-y)^{k} A(x, y) & =(x-y)^{k} B(x, y) \\
(z+y)^{l} A(z+y, y) & =(z+y)^{l} C(z, y) .
\end{aligned}
$$

In this way we try to eliminate the Jacobi identity and the notion of intertwining operator as it is usually defined.

Now, we can redefine the notion of tensor product with a ring theoretical interpretation:

Definition 4.4. Let $M$ and $N$ be two $V$-modules. A pair $\left(M \otimes N, F_{z}\right)$, which consists of a $V$-module $M \otimes_{V} N$ and a vertex bilinear map $F_{z}$ of type $\left(M, N ; M \otimes_{V} N\right)$, is called a tensor product for the ordered pair $(M, N)$ if the following universal property holds: For any $V$-module $W$ and any vertex bilinear map $I_{z}$ of type $(M, N ; W)$, there exists a unique $V$-homomorphism $\varphi$ from $M \underset{V}{\otimes} N$ to $W$ such that $I_{z}=\varphi \circ F_{z}$, where $\varphi$ is extended canonically to a linear map from $(M \underset{V}{\otimes} N)((z))$ to $W((z))$.

Using this universal property of the tensor product with respect to the vertex bilinear maps, we have:

Proposition 4.5. The space $V$ - bilinear $(M, N ; W)$ is linearly isomorphic to $\operatorname{Hom}_{V}\left(M \otimes_{V} N, W\right)$.

Now, we start our second (and equivalent) construction of the tensor product. Take $F_{1}(M, N)$ as before. Using the equivalence between the Jacobi identity with the identities (4.3) and (4.4), we can redefine $J_{1}$ as the $\mathfrak{g}(V)$-submodule of $F_{1}(M, N)$ linearly spanned by all the coefficients in the following expressions:

$$
\begin{gathered}
(z+x)^{N_{a, v}} a_{z \dot{+} x}\left(u \otimes_{x} v\right)-(z+x)^{N_{a, v}}\left(a_{\dot{z}} u{\underset{x}{x}}_{x} v\right) \\
(z-x)^{N_{a, u}} a_{\dot{z}}\left(u \otimes_{x} v\right)-(z-x)^{N_{a, u}}\left(u{\underset{x}{x}}_{\dot{z}} v\right) \\
\left(d u \otimes_{x} v\right)-\frac{d}{d x}\left(u{\underset{x}{\otimes} v) .}^{\otimes} v\right)
\end{gathered}
$$

for $a \in V, u \in M, v \in N$, and in this way we complete the picture of this ring theoretical construction.

This second construction is clearly the extension of the well known tensor product of modules over an associative commutative ring. 
Example 4.6. Let $V$ be an associative commutative algebra with unit. It is clear that the second construction of tensor product coincides with the usual tensor product of modules over a commutative ring.

Example 4.7. We may restrict our definitions and constructions to a graded vertex algebra $V$, and the category $C^{+}(V)$ of $\mathbb{Z}_{+^{-}}$graded $V$-modules, together with the intertwining operators that preserve the gradations (cf. p.132 [FZ] and p.56 [FHL]), in the sense that if $I_{z} \in\left(\begin{array}{c}W \\ M, N\end{array}\right)$ with

$$
I_{z}(u, v)=\sum_{n \in \mathbb{Z}} I_{(n)}(u, v) z^{-n-1},
$$

then for homogeneous elements $u \in M$ and $v \in N$, it satisfies

$$
I_{(n)}(u, v) \in W(\operatorname{deg} u+\operatorname{deg} v-n-1) .
$$

Since $W \in C^{+}(V)$, we have that

$$
I_{(k)}(u, v)=0 \quad \text { for all } \quad k \geq \operatorname{deg} u+\operatorname{deg} v,
$$

proving that pair $(M, N)$ satisfies the kernel intertwining operator full equality condition. Thus, there exists the tensor product of them, which is also $\mathbb{Z}_{+}$-graded by defining $\operatorname{deg}\left(t^{n} \otimes u \otimes v\right)=$ $\operatorname{deg}(u)+\operatorname{deg}(v)-n-1$.

In particular it holds for the category $C^{+}(V)$ of all known rational vertex operator algebras $V$ such as $V_{L}$, associated with a positive definite even lattice $L ; L(l, 0)$, associated with an affine Lie algebra with a positive integral level $l ; L\left(c_{p, q}, 0\right)$, associated with the Virasoro algebra with central charge $c_{p, q}$ in the minimal series; and $V^{\natural}$, the moonshine module.

Let $\operatorname{Mod}_{V}$ be the category of $V$-modules with the maps given by $\operatorname{Hom}_{V}(M, N)$ for $V$-modules $M$ and $N$. From now on we shall assume the existence of all the tensor product of modules that appear. One can restrict all the following results to certain subcategory where the tensor products always exist.

Proposition 4.8. Let $f: M \rightarrow M^{\prime}$ and $g: N \rightarrow N^{\prime}$ be homomorphisms of $V$-modules. Then there is a unique $V$-homomorphism denoted by $f \otimes g: M \underset{V}{\otimes} N \rightarrow M^{\prime} \underset{V}{\otimes} N^{\prime}$, with

$$
(f \otimes g)(u \underset{z}{\otimes} v)=f(u) \underset{z}{\otimes} g(v)
$$

for all $u \in M$ and $v \in N$.

Proof. The function $F_{z}: M \times N \rightarrow\left(M^{\prime} \otimes_{V} N^{\prime}\right)((z))$ given by $F_{z}(u, v)=f(u) \otimes_{z} g(v)$, is easily seen to be a vertex bilinear map. Then, by universal property, there is a unique $V$-homomorphism from $M \underset{V}{\otimes} N$ to $M^{\prime} \underset{V}{\otimes} N^{\prime}$ sending $u \underset{z}{\otimes} v$ to $f(u) \underset{z}{\otimes} g(v)$.

Corollary 4.9. Given $V$-homomorphisms $M \stackrel{f}{\rightarrow} M^{\prime} \stackrel{f^{\prime}}{\rightarrow} M^{\prime \prime}$ and $N \stackrel{g}{\rightarrow} N^{\prime} \stackrel{g^{\prime}}{\rightarrow} N^{\prime \prime}$, we have

$$
\left(f^{\prime} \otimes g^{\prime}\right)(f \otimes g)=f^{\prime} f \otimes g^{\prime} g .
$$

Proof. Both maps take $u \underset{z}{\otimes} v \mapsto f^{\prime}(f(u)) \underset{z}{\otimes} g^{\prime}(g(v))$, and so the uniqueness of such a homomorphism gives the desired equation.

Theorem 4.10. (a) Given $M$ a $V$-module, there is a covariant functor $F_{M}: \operatorname{Mod}_{V} \rightarrow \operatorname{Mod}_{V}$ defined by

for a $V$-homomorphism $g: N \rightarrow N^{\prime}$.

$$
F_{M}(N)=M \underset{V}{\otimes} N \quad \text { and } \quad F(g)=1_{M} \otimes g
$$


(b) Given $N$ a $V$-module, there is a covariant functor $G_{N}: \operatorname{Mod}_{V} \rightarrow \operatorname{Mod}_{V}$ defined by

$$
G_{N}(M)=M \underset{V}{\otimes} N \quad \text { and } \quad G(f)=f \otimes 1_{N}
$$

for a $V$-homomorphism $f: M \rightarrow M^{\prime}$.

Proof. First, note that $F_{M}$ preserves identities: $F_{M}\left(1_{N}\right)=1_{M} \otimes 1_{N}$ is the identity $1_{M \otimes N}$, because it fixes every generator. Second, $F_{M}$ preserves composition:

$$
F_{M}\left(g^{\prime} g\right)=1_{M} \otimes g^{\prime} g=\left(1_{M} \otimes g^{\prime}\right)\left(1_{M} \otimes g\right)=F_{M}\left(g^{\prime}\right) F_{M}(g)
$$

by Corollary 4.9. Therefore $F_{M}$ is a covariant functor. In a similar way one can prove (b).

We denote the functor $F_{M}$ by $M \underset{V}{\otimes} \square$, and the functor $G_{N}$ by $\square \underset{V}{\otimes} N$. We shall work with the tensor product "functor" when in fact we need to assume the existence.

\section{VERTEX HOMOMORPHISM AND ADJOINT ISOMORPHISMS}

In this section we introduce the notion of (right) vertex homomorphism, producing the "Hom" functor for modules over a vertex algebra, obtaining (from our point of view) an analog of the Hom functor for modules over an associative commutative algebra. We prefer to think about it in this way, instead of the Hom functor in Lie theory. In the first part of this section, we follow [Li1] and [Li2].

The most important functors in homological algebra are Hom, tensor product and functors derived from them. In the case of vertex algebras, we should consider Vhom (and later $V h o m^{r}$ ). We shall show that there is an intimate relationship between Vhom and tensor, they form an adjoint pair of functors.

Definition 5.1. Let $M$ and $N$ be two $V$-modules. A vertex homomorphism from $M$ to $N$ is a liner map $f_{z}: M \longrightarrow N((z))$ such that

(a) $f_{z}(d u)=\left(d-\frac{d}{d z}\right) f_{z}(u)$ for all $u \in M$.

(b) For any $a \in V$, there exists $k \in \mathbb{N}$ such that

$$
(z-x)^{k} a_{\dot{z}}^{N}\left(f_{x}(u)\right)=(z-x)^{k} f_{x}\left(a_{\dot{z}}^{M} u\right) \quad \text { for all } u \in M .
$$

We denote by $V h o m(M, N)$ the space of all vertex homomorphisms from $M$ to $N$. Define $d$ on $\operatorname{Vhom}(M, N)$ given by $(d f)_{z}=\frac{d}{d z}\left(f_{z}\right)$. Observe that if $V$ is an associative commutative algebra with unit and $d=0$, and $M$ and $N$ are modules over it with the corresponding derivations equal to zero, then (using (a) in the definition) any vertex homomorphism must be independent of $z$ and $\operatorname{Vhom}(M, N)=\operatorname{Hom}_{V}(M, N)$, the usual homomorphisms of modules over an associative commutative algebra.

One of the motivation for the definition of vertex homomorphism is the following: let $g_{z}$ : $M \times N \rightarrow W((z))$ be a vertex bilinear map. Then for any fixed $u \in M$, the map $f_{z}(v)=g_{z}(u, v)$ is a vertex homomorphism from $N$ to $W$.

Now, we define an action of $V$ on $\operatorname{Vhom}(M, N)$ as follows:

$$
\left(a_{\dot{z}} f\right)_{y}(u)=a_{z \dot{+} y}^{N}\left(f_{y}(u)\right)-f_{y}\left(a_{z \dot{+} y}^{M} u-a_{y \dot{+} z}^{M} u\right)
$$


which can also be rewritten as (cf. with associator formula (2.11))

$$
\left(a_{\dot{z}} f\right)_{y}(u)=\operatorname{Res}_{x}\left(\delta(x-y, z) a_{\dot{x}}^{N}\left(f_{y}(u)\right)-\delta(-y+x, z) f_{y}\left(a_{\dot{x}}^{M} u\right)\right),
$$

for $a \in V$ and $u \in M$.

Proposition 5.2. Let $a \in V, f \in V h o m(M, N)$ and $u \in M$. Then we have the following properties:

(a) $a_{\dot{z}} f \in \operatorname{Vhom}(M, N)((z))$.

(b) $\mathbf{1}_{\dot{z}} f=f$.

(c) $(d a)_{\dot{z}} f=\frac{d}{d z}\left(a_{\dot{z}} f\right)$.

(d) $d\left(a_{\dot{z}} f\right)=(d a)_{\dot{z}} f+a_{\dot{z}}(d f)$.

(e) For any integer $k$ such that $k \geq N_{a, u}$, we have

$$
(z+y)^{k}\left(a_{\dot{z}} f\right)_{y}(u)=(z+y)^{k} a_{z \dot{+} y}^{\stackrel{N}{N}}\left(f_{y}(u)\right) \in N((z, y)) .
$$

(f) For any integer $k$ such that $k \geq N_{a, u}$, an alternative definition for the action of $V$ in $\operatorname{Vhom}(M, N)$ is given by

$$
\left(a_{\dot{z}} f\right)_{y}(u)=(y+z)^{-k}\left((z+y)^{k} a_{z+\dot{+} y}^{N}\left(f_{y}(u)\right)\right) .
$$

similar to the ring theoretical case.

Proof. (a) If we write $\left(a_{\dot{z}} f\right)_{y}=\sum_{n \in \mathbb{Z}}\left(a_{n} f\right)_{y} z^{-n-1}$, then

$$
\left(a_{n} f\right)_{y}=\operatorname{Res}_{x}\left((x-y)^{n} a_{\dot{x}}^{N}\left(f_{y}\right)-(-y+x)^{n} f_{y}\left(a_{\dot{x}}^{M}\right)\right) .
$$

Therefore, there exists $N \in \mathbb{N}$ such that $\left(a_{n} f\right)_{y}=0$ for $n \geq N$.

Now, we should prove that $\left(a_{n} f\right)_{y}(d u)=\left(d-\frac{d}{d y}\right)\left(\left(a_{n} f\right)_{y}(u)\right)$ for all $n \in \mathbb{Z}$, or equivalently $\left(a_{\dot{z}} f\right)_{y}(d u)=\left(d-\frac{d}{d y}\right)\left(\left(a_{\dot{z}} f\right)_{y}(u)\right)$, that follows by

$$
\begin{aligned}
\frac{d}{d y}\left(\left(a_{\dot{z}} f\right)_{y}(u)\right)= & \frac{d}{d y} \operatorname{Res}_{x}\left[\delta(x-y, z) a_{\dot{x}}^{N}\left(f_{y}(u)\right)-\delta(-y+x, z) f_{y}\left(a_{\dot{x}}^{M} u\right)\right] \\
= & \operatorname{Res}_{x}\left[\left(\frac{d}{d y} \delta(x-y, z)\right) a_{\dot{x}}^{N}\left(f_{y}(u)\right)-\left(\frac{d}{d y} \delta(-y+x, z)\right) f_{y}\left(a_{\dot{x}}^{M} u\right)\right] \\
& +\operatorname{Res}_{x}\left[\delta(x-y, z) a_{\dot{x}}^{N}\left(\frac{d}{d y} f_{y}(u)\right)-\delta(-y+x, z) \frac{d}{d y}\left(f_{y}\left(a_{\dot{x}}^{M} u\right)\right)\right] \\
= & -\operatorname{Res}_{x}\left[\left(\frac{d}{d x} \delta(x-y, z)\right) a_{\dot{x}}^{N}\left(f_{y}(u)\right)-\left(\frac{d}{d x} \delta(-y+x, z)\right) f_{y}\left(a_{\dot{x}}^{M} u\right)\right] \\
& +\operatorname{Res}_{x}\left[\delta(x-y, z) a_{\dot{x}}^{N}\left(\frac{d}{d y} f_{y}(u)\right)-\delta(-y+x, z) \frac{d}{d y}\left(f_{y}\left(a_{\dot{x}}^{M} u\right)\right)\right] \\
= & \operatorname{Res}_{x}\left[\delta(x-y, z) \frac{d}{d x}\left(a_{\dot{x}}^{N}\left(f_{y}(u)\right)\right)-\delta(-y+x, z) f_{y}\left(\frac{d}{d x}\left(a_{\dot{x}}^{M} u\right)\right)\right] \\
& +\operatorname{Res}_{x}\left[\delta(x-y, z) a_{\dot{x}}^{N}\left(\frac{d}{d y} f_{y}(u)\right)-\delta(-y+x, z) \frac{d}{d y}\left(f_{y}\left(a_{\dot{x}}^{M} u\right)\right)\right]
\end{aligned}
$$




$$
\begin{aligned}
= & -\operatorname{Res}_{x}\left[\delta(x-y, z) a_{\dot{x}}^{N}\left(f_{y}(d u)\right)-\delta(-y+x, z) f_{y}\left(a_{\dot{x}}^{M}(d u)\right)\right] \\
& +\operatorname{Res}_{x}\left[\delta(x-y, z) d\left(a_{\dot{x}}^{N}\left(f_{y}(u)\right)\right)-\delta(-y+x, z) d\left(f_{y}\left(a_{\dot{x}}^{M} u\right)\right)\right] \\
= & d\left(\left(a_{\dot{z}} f\right)_{y}(u)\right)-\left(a_{\dot{z}} f\right)_{y}(d u) .
\end{aligned}
$$

Observe that (5.3) is the $(n)$-product of the fields $a_{\dot{y}}$ and $f_{y}$. Using that the pairs $\left(f_{y}, b_{\dot{y}}\right)$ and $\left(a_{\dot{y}}, b_{\dot{y}}\right)$ are local, together with Dong's lemma, we obtain that $b_{\dot{y}}$ and $\left(a_{n} f\right)_{y}$ are local. Thus $\left(a_{n} f\right)_{y} \in \operatorname{Vhom}(M, N)$, finishing (a). Part (b) is trivial.

(c) For $a \in V, u \in M, f \in \operatorname{Vhom}(M, N)$, we have

$$
\begin{aligned}
\left((d a)_{\dot{z}} f\right)_{y}(u) & =\operatorname{Res}_{x}\left(\delta(x-y, z)(d a)_{\dot{x}}^{N}\left(f_{y}(u)\right)-\delta(-y+x, z) f_{y}\left((d a)_{\dot{x}}^{M} u\right)\right) \\
& =\operatorname{Res}_{x}\left(\delta(x-y, z) \frac{d}{d x}\left(a_{\dot{x}}^{N}\left(f_{y}(u)\right)\right)-\delta(-y+x, z) \frac{d}{d x}\left(f_{y}\left(a_{\dot{x}}^{M} u\right)\right)\right) \\
& =\frac{d}{d z} \operatorname{Res}_{x}\left(\delta(x-y, z) a_{\dot{x}}^{N}\left(f_{y}(u)\right)-\delta(-y+x, z) f_{y}\left(a_{\dot{x}}^{M} u\right)\right)=\frac{d}{d z}\left(a_{\dot{z}} f\right)_{y}(u) .
\end{aligned}
$$

(d) For $a \in V, u \in M, f \in V h o m(M, N)$, we have

$$
\begin{aligned}
\left(d\left(a_{\dot{z}} f\right)\right)_{y}(u)-\left(a_{\dot{z}}(d f)\right)_{y}(u) & =\frac{d}{d y}\left(a_{\dot{z}} f\right)_{y}(u)-\left(a_{\dot{z}}(d f)\right)_{y}(u) \\
= & \frac{d}{d y} \operatorname{Res}_{x}\left(\delta(x-y, z) a_{\dot{x}}^{N}\left(f_{y}(u)\right)-\delta(-y+x, z) f_{y}\left(a_{\dot{x}}^{M} u\right)\right) \\
& -\operatorname{Res}_{x}\left(\delta(x-y, z) a_{\dot{x}}^{N}\left(\frac{d}{d y} f_{y}(u)\right)-\delta(-y+x, z) \frac{d}{d y} f_{y}\left(a_{\dot{x}}^{M} u\right)\right) \\
= & \operatorname{Res}_{x}\left(\left(\frac{d}{d y} \delta(x-y, z)\right)\left(a_{\dot{x}}^{N}\left(f_{y}(u)\right)\right)-\left(\frac{d}{d y} \delta(-y+x, z)\right) f_{y}\left(a_{\dot{x}}^{M} u\right)\right) \\
= & \frac{d}{d z}\left(\left(a_{\dot{z}} f\right)_{y}(u)\right)=\left((d a)_{\dot{z}} f\right)_{y}(u) .
\end{aligned}
$$

The proof of (e) and (f) is similar to the proof of (c) and (d) in Proposition 3.6.

Theorem 5.3. Vhom $(M, N)$ is a $V$-module.

Proof. We follow the proof of Theorem 6.1.7 in [Li2]. We only need to prove the Jacobi identity. For any $a, b \in V$ and $f \in V h o m(M, N)$, there exists $n \in \mathbb{N}$ such that

$$
\begin{aligned}
(y-z)^{n} a_{\dot{y}}^{N}\left(f_{z}\right) & =(y-z)^{n} f_{z}\left(a_{\dot{y}}^{M}\right) \\
(t-z)^{n} b_{\dot{t}}^{N}\left(f_{z}\right) & =(t-z)^{n} f_{z}\left(b_{\dot{t}}^{M}\right) \\
(y-t)^{n} a_{\dot{y}}^{M}\left(b_{\dot{t}}^{M}\right) & =(y-t)^{n} b_{\dot{t}}^{M}\left(a_{\dot{y}}^{M}\right) \\
(y-t)^{n} a_{\dot{y}}^{N}\left(b_{\dot{t}}^{N}\right) & =(y-t)^{n} b_{\dot{t}}^{N}\left(a_{\dot{y}}^{N}\right) .
\end{aligned}
$$

By definition, we have

$$
\begin{aligned}
\left(a_{\dot{w}}\left(b_{\dot{x}} f\right)\right)_{z} & =\operatorname{Res}_{y} \delta(y-z, w) a_{\dot{y}}^{N}\left(\left(b_{\dot{x}} f\right)_{z}\right)-\operatorname{Res}_{y} \delta(-z+y, w)\left(b_{\dot{y}} f\right)_{z}\left(a_{\dot{y}}^{M}\right) \\
& =\operatorname{Res}_{y} \operatorname{Res}_{t}(A-B-C+D),
\end{aligned}
$$


where

$$
\begin{aligned}
A & =\delta(y-z, w) \delta(t-z, x) a_{\dot{y}}^{N}\left(b_{\dot{t}}^{N}\left(f_{z}\right)\right) \\
B & =\delta(y-z, w) \delta(-z+t, x) a_{\dot{y}}^{N}\left(f_{z}\left(b_{\dot{t}}^{M}\right)\right) \\
C & =\delta(-z+y, w) \delta(t-z, x) b_{\dot{t}}^{N}\left(f_{z}\left(a_{\dot{y}}^{M}\right)\right) \\
D & =\delta(-z+y, w) \delta(-z+t, x) f_{z}\left(b_{\dot{t}}^{M}\left(a_{\dot{y}}^{M}\right)\right) .
\end{aligned}
$$

Similarly, we have

$$
\left(b_{\dot{x}}\left(a_{\dot{w}} f\right)\right)_{z}=\operatorname{Res}_{y} \operatorname{Res}_{t}\left(A^{\prime}-B^{\prime}-C^{\prime}+D^{\prime}\right),
$$

where

$$
\begin{aligned}
A^{\prime} & =\delta(y-z, w) \delta(t-z, x) b_{\dot{t}}^{N}\left(a_{\dot{y}}^{N}\left(f_{z}\right)\right) \\
B^{\prime} & =\delta(y-z, w) \delta(-z+t, x) b_{\dot{t}}^{N}\left(f_{z}\left(a_{\dot{y}}^{M}\right)\right) \\
C^{\prime} & =\delta(-z+y, w) \delta(t-z, x) a_{\dot{y}}^{N}\left(f_{z}\left(b_{\dot{t}}^{M}\right)\right) \\
D^{\prime} & =\delta(-z+y, w) \delta(-z+t, x) f_{z}\left(a_{\dot{y}}^{M}\left(b_{\dot{t}}^{M}\right)\right) .
\end{aligned}
$$

By the properties of the $\delta$-function, we obtain

$$
\delta(w-x, r) r^{n} w^{n} x^{n} Q=\delta(w-x, r)(y-z)^{n}(t-z)^{n}(y-t)^{n} Q
$$

for any $Q \in\{A, B, C, D\}$. Therefore

$$
\begin{aligned}
\delta(w-x, r) r^{n} w^{n} x^{n}(A-B-C+D) & =\delta(w-x, r)(y-z)^{n}(t-z)^{n}(y-t)^{n}(A-B-C+D) \\
& =\operatorname{Res}_{y} \operatorname{Res}_{t} \delta(w-x, r) \delta(y-w, z) \delta(t-x, z) X,
\end{aligned}
$$

where

Similarly, we have

$$
X=(y-z)^{n}(t-z)^{n}(y-t)^{n} a_{\dot{y}}^{N}\left(b_{\dot{t}}^{N}\left(f_{z}\right)\right) .
$$

$\delta(-x+w, r) r^{n} w^{n} x^{n}\left(A^{\prime}-B^{\prime}-C^{\prime}+D^{\prime}\right)=\operatorname{Res}_{y} \operatorname{Res}_{t} \delta(-x+w, r) \delta(y-w, z) \delta(t-x, z) X$.

Thus

$$
\begin{aligned}
r^{n} w^{n} x^{n}\left[\left(\delta(w-x, r) a_{\dot{w}}\left(b_{\dot{x}}\right)-\right.\right. & \left.\left.\delta(-x+w, r) b_{\dot{x}}\left(a_{\dot{w}}\right)\right) f\right]_{z} \\
= & \operatorname{Res}_{y} \operatorname{Res}_{t} \delta(w-r, x) \delta(y-w, z) \delta(t-x, z) X .
\end{aligned}
$$

On the other hand, we have by definition

$$
\begin{aligned}
& r^{n} w^{n} x^{n} \delta(w-r, x)\left(\left(a_{\dot{r}} b\right)_{\dot{x}} f\right)_{z}= \\
& \quad=\operatorname{Res}_{t} r^{n} w^{n} x^{n} \delta(w-r, x)\left(\delta(t-z, x)\left(a_{\dot{r}} b\right)_{\dot{t}}^{N}\left(f_{z}\right)-\delta(-z+t, x) f_{z}\left(\left(a_{\dot{r}} b\right)_{\dot{t}}^{M}\right)\right) .
\end{aligned}
$$

But, using that for $W=M$ or $W=N$ we have:

$$
\begin{aligned}
r^{n}\left(a_{\dot{r}} b\right)_{\dot{t}}^{W} & =\operatorname{Res}_{y} r^{n}\left(\delta(y-t, r) a_{\dot{y}}^{W}\left(b_{\dot{t}}^{W}\right)-\delta(-t+y, r) b_{\dot{t}}^{W}\left(a_{\dot{y}}^{W}\right)\right) \\
& =\operatorname{Res}_{y}\left(\delta(y-t, r)(y-t)^{n} a_{\dot{y}}^{W}\left(b_{\dot{t}}^{W}\right)-\delta(-t+y, r)(y-t)^{n} b_{\dot{t}}^{W}\left(a_{\dot{y}}^{W}\right)\right) \\
& =\operatorname{Res}_{y} \delta(y-r, t)(y-t)^{n} a_{\dot{y}}^{W}\left(b_{\dot{t}}^{W}\right),
\end{aligned}
$$


then, we obtain

$$
\begin{aligned}
& r^{n} w^{n} x^{n} \delta(w-r, x)\left(\left(a_{\dot{r}} b\right)_{\dot{x}} f\right)_{z}= \\
& =\operatorname{Res}_{t} \operatorname{Res}_{y} \delta(w-r, x) \delta(t-z, x) \delta(y-r, t) X-\operatorname{Res}_{t} \operatorname{Res}_{y} \delta(w-r, x) \delta(-z+t, x) \delta(y-r, t) X \\
& =\operatorname{Res}_{t} \operatorname{Res}_{y} \delta(w-r, x) \delta(t-x, z) \delta(y-r, t) X
\end{aligned}
$$

By using the properties of $\delta$, we obtain that the product of the $\delta$ 's in (5.5) is

$$
\begin{aligned}
& \delta(w-r, x) \delta(t-x, z) \delta(y-r, t)=\delta(w-r, x) \delta(z+x, t) \delta(t+r, y)= \\
& =\delta(w-r, x) \delta(z+x, t) \delta((z+x)+r, y)=\delta(w-r, x) \delta(z+x, t) \delta(z+w, y)= \\
& =\delta(w-r, x) \delta(t-x, z) \delta(y-w, z) .
\end{aligned}
$$

Therefore, using (5.4) and (5.5), we have

$$
r^{n} w^{n} x^{n} \delta(w-r, x)\left(\left(a_{\dot{r}} b\right)_{\dot{x}} f\right)_{z}=r^{n} w^{n} x^{n}\left[\left(\delta(w-x, r) a_{\dot{w}}\left(b_{\dot{x}}\right)-\delta(-x+w, r) b_{\dot{x}}\left(a_{\dot{w}}\right)\right) f\right]_{z}
$$

Multiplying both sides by $r^{-n} w^{-n} x^{-n}$, we obtain the Jacobi identity.

Let $M, M^{\prime}, N$ be $V$-modules. Given $f \in \operatorname{Hom}_{V}\left(M^{\prime}, M\right)$ we define the induced map

$$
f_{*}: \operatorname{Hom}_{V}\left(N, M^{\prime}\right) \rightarrow \operatorname{Hom}_{V}(N, M)
$$

given by $\left[f_{*}(\psi)\right](v)=f(\psi(v))$ for all $v \in N$. Similarly, we define

$$
f^{*}: \operatorname{Hom}_{V}(M, N) \rightarrow \operatorname{Hom}_{V}\left(M^{\prime}, N\right)
$$

given by $\left[f^{*}(\phi)\right]\left(u^{\prime}\right)=\phi\left(f\left(u^{\prime}\right)\right)$ for all $u^{\prime} \in M^{\prime}$. By standard arguments one can see that $\operatorname{Hom}_{V}(N, \square)$ (resp. $\operatorname{Hom}_{V}(\square, N)$ ) is a covariant (resp. contravariant) functor from Mod $\mathrm{M}_{V}$ to Vect $_{\mathbf{k}}$ (the category of $\mathbf{k}$-vector spaces).

Now, we are interested in $V h o m$. Let $M, M^{\prime}$ and $N$ be $V$-modules. Given $f \in \operatorname{Hom}_{V}\left(M^{\prime}, M\right)$, we can define an induced map

$$
f_{*}: \operatorname{Vhom}\left(N, M^{\prime}\right) \rightarrow \operatorname{Vhom}(N, M)
$$

given by $\left[f_{*}(\psi)\right]_{z}(v)=f\left(\psi_{z}(v)\right)$ for all $v \in N$. Similarly, we define

$$
f^{*}: \operatorname{Vhom}(M, N) \rightarrow \operatorname{Vhom}\left(M^{\prime}, N\right)
$$

given by $\left[f^{*}(\phi)\right]_{z}\left(u^{\prime}\right)=\phi_{z}\left(f\left(u^{\prime}\right)\right)$ for all $u^{\prime} \in M^{\prime}$. A simple computation shows that $f_{*}(\psi) \in$ $\operatorname{Vhom}(N, M)$ and $f^{*}(\phi) \in \operatorname{Vhom}\left(M^{\prime}, N\right)$.

Observe that we use the same notation $f_{*}$ and $f^{*}$ for Vhom and $\mathrm{Hom}_{V}$.

Proposition 5.4. (a) The maps $f_{*}$ and $f^{*}$ are $V$-homomorphisms.

(b) $\operatorname{Vhom}(N, \square)$ is a covariant functor from $\operatorname{Mod}_{V}$ to $\operatorname{Mod}_{V}$.

(c) Vhom $(\square, N)$ is a contravariant functor from $\operatorname{Mod}_{V}$ to $\operatorname{Mod}_{V}$.

Proof. (a) It is clear that $f_{*}$ is additive, and

$$
\left[f_{*}(d \psi)\right]_{z}(v)=f\left((d \psi)_{z}(v)\right)=\frac{d}{d z} f\left(\psi_{z}(v)\right)=\left[d\left(f_{*}(\psi)\right)\right]_{z}(v) .
$$

So, we should check that $f_{*}\left(a_{\dot{z}} \psi\right)=a_{\dot{z}}\left[f_{*}(\psi)\right]$ for any $a \in V$. But, for all $v \in N$, we have

$$
\left[f_{*}\left(a_{\dot{z}} \psi\right)\right]_{x}(v)=f\left(\left(a_{\dot{z}} \psi\right)_{x}(v)\right)=f\left(a_{z \dot{+} x}\left(\psi_{x}(v)\right)-\psi_{x}\left(a_{z \dot{+} x} v-a_{x \dot{+} z} v\right)\right) \text {. }
$$


On the other hand, we have

$$
\begin{aligned}
\left(a_{\dot{z}}\left[f_{*}(\psi)\right]\right)_{x}(v) & =a_{z \dot{+} x}\left(\left[f_{*}(\psi)\right]_{x}(v)\right)-\left[f_{*}(\psi)\right]_{x}\left(a_{z \dot{+} x} v-a_{x \dot{+} z} v\right) \\
& =a_{z \dot{+} x}\left(f\left(\psi_{x}(v)\right)\right)-f\left(\psi_{x}\left(a_{z \dot{+} x} v-a_{x \dot{+} z} v\right)\right) \\
& =f\left(a_{z \dot{+} x}\left(\psi_{x}(v)\right)\right)-f\left(\psi_{x}\left(a_{z \dot{+} x} v-a_{x \dot{+} z} v\right)\right),
\end{aligned}
$$

proving that $f_{*}$ is a $V$-homomorphism. Similarly, by a simple computation, one can check that $f^{*}$ is also a $V$-homomorphism.

By standard computations one can see that these are functors, proving (b) and (c). For example, if $f \in \operatorname{Hom}_{V}\left(M^{\prime}, M\right)$ and $g \in \operatorname{Hom}_{V}\left(M, M^{\prime \prime}\right)$, then for $\psi \in \operatorname{Vhom}_{V}\left(N, M^{\prime}\right)$ we have

$$
\left[g_{*}\left(f_{*}(\psi)\right)\right]_{z}(v)=g\left(\left[f_{*}(\psi)\right]_{z}(v)\right)=g\left(f\left(\psi_{z}(v)\right)\right)=(g f)\left(\psi_{z}(v)\right)=\left[(g f)_{*}(\psi)\right]_{z}(v),
$$

for all $v \in N$.

Now, we shall present some properties of Vhom (cf. Proposition 6.2.5 in [Li2]).

Proposition 5.5. Let $M$ be a $V$-module. Then $\operatorname{Vhom}(V, M)$ is isomorphic to $M$ as a $V$ module. The isomorphism is given by $\Psi_{M}: \operatorname{Vhom}(V, M) \rightarrow M$ with $\Psi_{M}(f)=\operatorname{Res}_{z} z^{-1} f_{z}(\mathbf{1})$. Moreover, if $g \in \operatorname{Hom}_{V}(M, N)$, then the following diagram commutes:

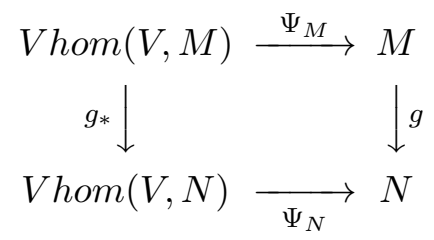

proving that $\Psi$ is a natural isomorphism from $\operatorname{Vhom}(V, \square)$ to the identity functor on $\operatorname{Mod}_{V}$.

Proof. We define a map

$$
\Phi: M \longrightarrow \operatorname{Vhom}(V, M)
$$

given by $[\Phi(u)]_{z}(a)=e^{z d}\left(a_{-z} u\right)$, for $u \in M$. We should check that $[\Phi(u)]_{z} \in \operatorname{Vhom}(V, M)$. By definition, we have

$$
\begin{aligned}
{[\Phi(u)]_{z}(d a) } & =-e^{z d}\left(\frac{d}{d z}\left(a_{-z} u\right)\right)=\left(\frac{d}{d z} e^{z d}\right)\left(a_{-z} u\right)-\frac{d}{d z}\left(e^{z d}\left(a_{-z} u\right)\right) \\
& =\left(d-\frac{d}{d z}\right)\left(e^{z d}\left(a_{-z} u\right)\right)=\left(d-\frac{d}{d z}\right)[\Phi(u)]_{z}(a) .
\end{aligned}
$$

Using associativity, for any $b \in V$, there is $k \in \mathbb{N}$ depending only on $b$ and $u$ (see Remark 2.4) such that

$$
(z+x)^{k} b_{z \dot{+} x}\left(a_{\dot{x}} u\right)=(z+x)^{k}\left(b_{\dot{z}} a\right)_{\dot{x}} u
$$

for all $a \in V$. Thus,

$$
(z+x)^{k} b_{z \dot{+} x}\left(e^{x d}[\Phi(u)]_{-x}(a)\right)=(z+x)^{k} e^{x d}\left([\Phi(u)]_{-x}\left(b_{\dot{z}} a\right)\right) .
$$

Using conjugation by $e^{x d}$, we get $(z+x)^{k} b_{\dot{z}}\left([\Phi(u)]_{-x}(a)\right)=(z+x)^{k}[\Phi(u)]_{-x}\left(b_{\dot{z}} a\right)$ for all $a \in V$, proving that $[\Phi(u)]_{z} \in \operatorname{Vhom}(V, M)$. It is easy to see using the commutator formula for modules (2.12) that the linear map $\Phi$ from $M$ to $\operatorname{Vhom}(V, M)$ is a $V$-homomorphism.

Conversely, given any $f_{z} \in V h o m(V, M)$, if we write $f_{z}=\sum_{n \in \mathbb{Z}} f_{n} z^{-n-1}$, then by definition $f_{n}(d a)=d\left(f_{n}(a)\right)+n f_{n-1}(a)$. In particular,

$$
d\left(f_{n}(\mathbf{1})\right)=-n f_{n-1}(\mathbf{1}) .
$$


But, $f_{z}(\mathbf{1})$ is a Laurent serie, hence $f_{m}(\mathbf{1})=0$ for some nonnegative $m$. Therefore, it follows that $f_{n}(\mathbf{1})=0$ for all nonnegative $n$. Thus, $f_{z}(\mathbf{1})$ involves only nonnegative powers of $z$, and using (5.6), one can easily get that $f_{z}(\mathbf{1})=e^{z d} u$, where $u=\Psi_{M}(f)=f_{-1}(\mathbf{1}) \in M$. Then

$$
\begin{aligned}
\Psi_{M}\left(a_{\dot{x}} f\right) & =\operatorname{Res}_{z} z^{-1}\left(a_{\dot{x}} f\right)_{z}(\mathbf{1})=\operatorname{Res}_{z} z^{-1} a_{x \dot{+} z}\left(f_{z}(\mathbf{1})\right)=\operatorname{Res}_{z} z^{-1} a_{x \dot{+} z}\left(e^{z d} u\right) \\
& =a_{\dot{x}} u=a_{\dot{x}}\left(\Psi_{M}(f)\right),
\end{aligned}
$$

proving that $\Psi_{M}$ is a $V$-homomorphism. Now, for any $a \in V$ there exists $k \in \mathbb{N}$ such that $(z-x)^{k} f_{z}\left(a_{\dot{x}}\right)=(z-x)^{k} a_{\dot{x}}\left(f_{z}\right)$. Then

$$
\begin{aligned}
z^{k} f_{z}(a) & =(z-x)^{k} f_{z}\left(a_{\dot{x}} \mathbf{1}\right)_{\left.\right|_{x=0}}=(z-x)^{k} a_{\dot{x}}\left(f_{z}(\mathbf{1})\right)_{\left.\right|_{x=0}}=(z-x)^{k} a_{\dot{x}}\left(e^{z d} u\right)_{\left.\right|_{x=0}} \\
& =(z-x)^{k} e^{z d}\left(a_{x \dot{z} z} u\right)_{\left.\right|_{x=0}}=z^{k} e^{z d}\left(a_{-\dot{z}} u\right) .
\end{aligned}
$$

Thus, $f=\Phi\left(\Psi_{M}(f)\right)$. Therefore $\operatorname{Vhom}(V, M)$ is isomorphic to $M$ as a $V$-module. Finally, we have

$$
\Psi_{N}\left(g_{*}(\psi)\right)=\operatorname{Res}_{z} z^{-1}\left[g_{*}(\psi)\right]_{z}(\mathbf{1})=\operatorname{Res}_{z} z^{-1} g\left(\psi_{z}(\mathbf{1})\right)=g\left(\Psi_{M}(\psi)\right),
$$

finishing the proof.

The intertwining operators of type $\left({ }_{M}^{W}{ }_{N}\right)$ are usually related to $\operatorname{Hom}_{V}(M, \operatorname{Vhom}(N, W))$ and $\operatorname{Hom}_{V}\left(N, \operatorname{Vhom}^{r}(M, W)\right)$ as in [Li1] and [DLM]. But we shall eliminate the notion of intertwining operator in the statement of Theorem 5.6 (and Theorem 5.14 for $V h^{\prime} m^{r}$ ), following the ring theoretical point of view. The following result could be thought as a motivation for the definition of $V h o m$ and the action of $V$ on it.

Theorem 5.6. (Adjoint isomorphism, first version) Let $M, N$ and $W$ be $V$-modules. Then there is a natural isomorphism of $\mathbf{k}$-vector spaces

$$
\tau_{M, N, W}: \operatorname{Hom}_{V}(M \underset{V}{\otimes} N, W) \longrightarrow \operatorname{Hom}_{V}(M, \operatorname{Vhom}(N, W))
$$

sending $f: M \underset{V}{\otimes} N \rightarrow W$ to

$$
\left[\tau_{M, N, W}(f)\right](u): v \mapsto f(u \underset{z}{\otimes} v)
$$

for $u \in M$ and $v \in N$. In more detail, fixing any two of $M, N, W$, each $\tau_{M, N, W}$ is a natural isomorphism:

$$
\begin{gathered}
\operatorname{Hom}_{V}(\square \underset{V}{\otimes} N, W) \longrightarrow \operatorname{Hom}_{V}(\square, \operatorname{Vhom}(N, W)) \\
\operatorname{Hom}_{V}(M \underset{V}{\otimes} \square, W) \longrightarrow \operatorname{Hom}_{V}(M, \operatorname{Vhom}(\square, W)) \\
\operatorname{Hom}_{V}\left(M{\underset{V}{V}}_{\mathbb{N}}^{\otimes} N, \square\right) \longrightarrow \operatorname{Hom}_{V}(M, \operatorname{Vhom}(N, \square)) .
\end{gathered}
$$

For example, if $f \in \operatorname{Hom}_{V}\left(M, M^{\prime}\right)$, then the following diagram commutes:

$$
\begin{array}{cl}
\operatorname{Hom}_{V}\left(M^{\prime} \underset{V}{\otimes} N, W\right) \stackrel{\tau_{M^{\prime}, N, W}}{\longrightarrow} \operatorname{Hom}_{V}\left(M^{\prime}, \operatorname{Vhom}(N, W)\right) \\
\left(f \otimes 1_{N}\right)^{*} \downarrow \\
\operatorname{Hom}_{V}(M \underset{V}{\otimes} N, W) \underset{\tau_{M, N, W}}{\longrightarrow} \operatorname{Hom}_{V}(M, \operatorname{Vhom}(N, W))
\end{array}
$$

Proof. (cf. Theorem 7.2.1 in [Li1]) Using Proposition 4.5, we have that $\operatorname{Hom}_{V}(M \otimes N, W)$ is linearly isomorphic (in a natural way) to $V$-Bilinear $(M, N ; W)$, practically by definition. It remains to show that

$$
V \text { - Bilinear }(M, N ; W) \simeq \operatorname{Hom}_{V}(M, \operatorname{Vhom}(N, W)) .
$$


Let $\phi \in \operatorname{Hom}_{V}(M, \operatorname{Vhom}(N, W))$. Then we define a k-bilinear map $F_{z}^{\phi}: M \times N \rightarrow W((z))$ given by

By definition, we have

$$
F_{z}^{\phi}(u, v)=[\phi(u)]_{z}(v) \quad \text { for } u \in M, v \in N
$$

$$
F_{z}^{\phi}(d u, v)=[\phi(d u)]_{z}(v)=[d \phi(u)]_{z}(v)=\frac{d}{d z}[\phi(u)]_{z}(v)=\frac{d}{d z} F_{z}^{\phi}(u, v)
$$

and

$$
F_{z}^{\phi}(u, d v)=[\phi(u)]_{z}(d v)=\left(d-\frac{d}{d z}\right)[\phi(u)]_{z}(v)=\left(d-\frac{d}{d z}\right) F_{z}^{\phi}(u, v) .
$$

Furthermore, for $a \in V, u \in M$ and $v \in N$, by the action on $V h o m$ (5.2), we have

$$
\begin{aligned}
F_{x}^{\phi}\left(a_{\dot{z}} u, v\right) & =\left[\phi\left(a_{\dot{z}} u\right)\right]_{x}(v)=\left[a_{\dot{z}} \phi(u)\right]_{x}(v)=a_{z \dot{+} x}\left([\phi(u)]_{x}(v)\right)-[\phi(u)]_{x}\left(a_{z \dot{+} x} v-a_{x \dot{+} z} v\right) \\
& =a_{z \dot{+} x}\left(F_{x}^{\phi}(u, v)\right)-F_{x}^{\phi}\left(u, a_{z \dot{+} x} v-a_{x \dot{+} z} v\right) .
\end{aligned}
$$

Thus, for all $a \in V$ and $v \in N$, there exists $n \in \mathbb{N}\left(n \geq N_{a, v}\right)$ such that

$$
(z+x)^{n} F_{x}^{\phi}\left(a_{\dot{z}} u, v\right)=(z+x)^{n} a_{z \dot{+} x} F_{x}^{\phi}(u, v), \quad \text { for all } u \in M .
$$

Using (b) in the definition of $V h o m$, for all $a \in V$ and $u \in M$ there exists $k \in \mathbb{N}$ such that

$$
(z-x)^{k} F_{x}^{\phi}\left(u, a_{\dot{z}} v\right)=(z-x)^{k} a_{\dot{z}}\left(F_{x}^{\phi}(u, v)\right) \quad \text { for all } v \in N .
$$

Therefore, using Definition 4.1.(3), we have that $F_{x}^{\phi}$ is a vertex bilinear map of type $(M, N ; W)$, obtaining a linear map from $\operatorname{Hom}_{V}(M, \operatorname{Vhom}(N, W))$ to $V$ - Bilinear $(M, N ; W)$.

Conversely, for any $F_{z} \in V$-Bilinear $(M, N ; W)$, it is clear by Definition 4.1.(3), that for each $u \in M$, we have $F_{z}(u, \cdot) \in \operatorname{Vhom}(N, W)$. Then we obtain a linear map $\phi^{F}: M \rightarrow \operatorname{Vhom}(N, W)$ (compare it with $\tau_{M, N, W}$ ) defined by

$$
\left[\phi^{F}(u)\right]_{z}(v)=F_{z}(u, v) .
$$

For any $a \in V$ and $u \in M$, using the associator formula (2.17) and the action on Vhom (5.2), we get

$$
\left[\phi^{F}\left(a_{\dot{z}} u\right)\right]_{x}(v)=F_{x}\left(a_{\dot{z}} u, v\right)=a_{z \dot{+} x} F_{x}(u, v)-F_{x}\left(u, a_{z \dot{+} x} v-a_{x \dot{+} z} v\right)=\left[a_{\dot{z}} \phi^{F}(u)\right]_{x}(v) .
$$

Therefore $\phi^{F} \in \operatorname{Hom}_{V}(M, \operatorname{Vhom}(N, W))$, proving the isomorphism.

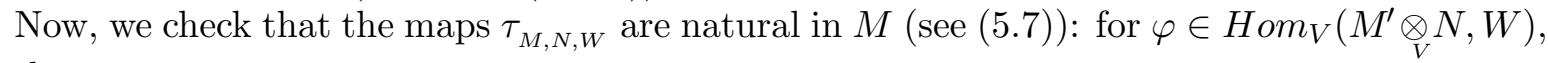
we have

$$
\begin{aligned}
{\left[\left(\tau_{M, N, W}\left[\left(f \otimes 1_{N}\right)^{*}(\varphi)\right]\right)(u)\right]_{z}(v) } & =\left[\left(f \otimes 1_{N}\right)^{*}(\varphi)\right](u \underset{z}{\otimes} v)=\varphi\left(\left(f \otimes 1_{N}\right)(u \underset{z}{\otimes} v)\right) \\
& =\varphi(f(u) \underset{z}{\otimes} v),
\end{aligned}
$$

and

$$
\left(\left[f^{*}\left(\tau_{M, N, W}(\varphi)\right)\right](u)\right)_{z}(v)=\left(\left[\tau_{M^{\prime}, N, W}(\varphi)\right](f(u))\right)_{z}(v)=\varphi(f(u) \underset{z}{\otimes} v)
$$

proving naturality in $M$. One can check that the other cases are natural by standard arguments.

In the next part of the work, we follow in part [DLM]. 
Definition 5.7. Let $M$ and $N$ be two $V$-modules. A right vertex homomorphism from $M$ to $N$ is a liner map $f_{z}: M \longrightarrow N((z))$ such that

(a) $f_{z}(d u)=\frac{d}{d z} f_{z}(u)$ for all $u \in M$.

(b) For any $a \in V$, there exists $k \in \mathbb{N}$ such that

$$
(z+x)^{k} a_{z+x}\left(f_{x}(u)\right)=(z+x)^{k} f_{x}\left(a_{\dot{z}} u\right) \quad \text { for all } u \in M .
$$

Observe that in [DLM] Definition 2.11, they give a similar notion, but the integer $k$ in (b) also depends on $u \in M$, which is wrong.

We denote by $\operatorname{Vhom}^{r}(M, N)$ the space of all right vertex homomorphisms from $M$ to $N$. Define $d$ on $\operatorname{Vhom}^{r}(M, N)$ given by $(d f)_{z}(u)=\left(d-\frac{d}{d z}\right)\left(f_{z}(u)\right)$, for $u \in M$. Observe that if $V$ is an associative commutative algebra with unit and $d=0$, and $M$ and $N$ are modules over it with the corresponding derivations equal to zero, then (using (a) in the definition) any right vertex homomorphism must be independent of $z$ and $\operatorname{Vhom}^{r}(M, N)=\operatorname{Hom}_{V}(M, N)$, the usual homomorphisms of modules over an associative commutative algebra.

One of the motivation for the definition of right vertex homomorphism is the following: let $g_{z}: M \times N \rightarrow W((z))$ be a vertex bilinear map. Then for any fixed $v \in N$, the map $f_{z}(u)=$ $g_{z}(u, v)$ is a right vertex homomorphism from $M$ to $W$.

Now, we define an action of $V$ on $\operatorname{Vhom}^{r}(M, N)$ as follows:

$$
\left(a_{\dot{z}}^{r} f\right)_{x}(u)=a_{\dot{z}}\left(f_{x}(u)\right)-f_{x}\left(a_{z \dot{-} x} u-a_{-\dot{x}+z} u\right)
$$

which can also be rewritten as

$$
\left(a_{\dot{z}}^{r} f\right)_{x}(u)=a_{\dot{z}}\left(f_{x}(u)\right)-\operatorname{Res}_{y} \delta(x+y, z) f_{w}\left(a_{\dot{y}} u\right),
$$

for $a \in V$ and $u \in M$.

Similarly, we can prove (see Proposition 5.2 (e) and (f)):

Proposition 5.8. Let $a \in V, f \in \operatorname{Vhom}^{r}(M, N)$ and $u \in M$. Then we have the following properties:

(a) For any integer $k$ such that $k \geq N_{a, u}$, we have

$$
(z-x)^{k}\left(a_{\dot{z}}^{r} f\right)_{x}(u)=(z-x)^{k} a_{\dot{z}}\left(f_{x}(u)\right) \in N((z, x)) .
$$

(b) For any integer $k$ such that $k \geq N_{a, u}$, an alternative definition for the action of $V$ in $\operatorname{Vhom}^{r}(M, N)$ is given by

$$
\left(a_{\dot{z}}^{r} f\right)_{x}(u)=(-x+z)^{-k}\left((z-x)^{k} a_{\dot{z}}\left(f_{x}(u)\right)\right) .
$$

similar to the ring theoretical case.

Now, we define $T$ as the operator in $\operatorname{Hom}_{\mathbf{k}}(M, N((z)))$ given by

$$
[T(f)]_{z}(u)=e^{z d} f_{-z}(u),
$$

for any $f \in \operatorname{Hom}_{\mathbf{k}}(M, N((z)))$ and $u \in M$. Then we have the following result (cf. Proposition 2.12 in $[\mathrm{DLM}])$.

Proposition 5.9. Let $f \in \operatorname{Hom}_{\mathbf{k}}(M, N((z)))$. Then $f \in \operatorname{Vhom}(M, N)$ if and only if $T(f) \in$ $\operatorname{Vhom}^{r}(M, N)$. 
Proof. For $u \in M$, by definition we have

$$
\begin{aligned}
\frac{d}{d z}[T(f)]_{z}(u) & =d e^{z d} f_{-z}(u)+e^{z d} \frac{d}{d z}\left(f_{-z}(u)\right) \\
& =e^{z d}\left(d f_{-z}(u)-f_{-z}(d u)+\frac{d}{d z}\left(f_{-z}(u)\right)\right)+[T(f)]_{z}(d u) .
\end{aligned}
$$

Then $\frac{d}{d z}[T(f)]_{z}(u)=[T(f)]_{z}(d u)$ if and only if $\left(d-\frac{d}{d z}\right) f_{z}(u)=f_{z}(d u)$.

For any $a \in V$, suppose that there exists $k \in \mathbb{N}$ such that

$$
(z+x)^{k} a_{z \dot{+} x}\left([T(f)]_{x}(u)\right)=(z+x)^{k}[T(f)]_{x}\left(a_{\dot{z}} u\right) \quad \text { for all } u \in M .
$$

Then

$$
(z+x)^{k} e^{-x d} a_{z \dot{+} x}\left([T(f)]_{x}(u)\right)=(z+x)^{k} e^{-x d}[T(f)]_{x}\left(a_{\dot{z}} u\right) .
$$

Thus

That is,

$$
(z+x)^{k} a_{\dot{z}}\left(e^{-x d}[T(f)]_{x}(u)\right)=(z+x)^{k} e^{-x d}[T(f)]_{x}\left(a_{\dot{z}} u\right) .
$$

$$
(z+x)^{k} a_{\dot{z}}\left(f_{-x}(u)\right)=(z+x)^{k} f_{-x}\left(a_{\dot{z}} u\right) .
$$

Since every step in the proof can be reversed, $f$ satisfies (5.1) if and only if $T(f)$ satisfies (5.8).

Proposition 5.10. (cf. Proposition 2.13 in $[\mathrm{DLM}]$ ) For any $a \in V$ and $f \in \operatorname{Vhom}(M, N)$, we have

$$
T\left(a_{\dot{z}} f\right)=a_{\dot{z}}^{r}[T(f)]
$$

Proof. By definition we have

$$
\begin{aligned}
{\left[T\left(a_{\dot{z}} f\right)\right]_{x}(u) } & =e^{x d}\left(a_{z \dot{-x}}\left(f_{-x}(u)\right)-f_{-x}\left(a_{z \dot{-x}} u-a_{-\dot{x}+z} u\right)\right) \\
& =a_{\dot{z}}\left(e^{x d} f_{-x}(u)\right)-e^{x d} f_{-x}\left(a_{z \dot{-x}} u-a_{-\dot{x}+z} u\right) \\
& =a_{\dot{z}}\left([T(f)]_{x}(u)\right)-[T(f)]_{x}\left(a_{z-x} u-a_{-\dot{x}+z} u\right) \\
& =\left(a_{\dot{z}}^{r}[T(f)]\right)_{x}(u) .
\end{aligned}
$$

Then we obtain

Proposition 5.11. $\operatorname{Vhom}^{r}(M, N)$ is a $V$-module and the linear map $T$ is a $V$-isomorphism.

Let $M, M^{\prime}$ and $N$ be $V$-modules. Given $f \in H \operatorname{mom}_{V}\left(M^{\prime}, M\right)$, we can define an induced map

$$
f_{*}: \operatorname{Vhom}^{r}\left(N, M^{\prime}\right) \rightarrow \operatorname{Vhom}^{r}(N, M)
$$

given by $\left[f_{*}(\psi)\right]_{z}(v)=f\left(\psi_{z}(v)\right)$ for all $v \in N$. Similarly, we define

$$
f^{*}: \operatorname{Vhom}^{r}(M, N) \rightarrow \operatorname{Vhom}^{r}\left(M^{\prime}, N\right)
$$

given by $\left[f^{*}(\phi)\right]_{z}\left(u^{\prime}\right)=\phi_{z}\left(f\left(u^{\prime}\right)\right)$ for all $u^{\prime} \in M^{\prime}$. A simple computation shows that $f_{*}(\psi) \in$ $\operatorname{Vhom}^{r}(N, M)$ and $f^{*}(\phi) \in \operatorname{Vhom}^{r}\left(M^{\prime}, N\right)$.

Observe that we use the same notation of $f_{*}$ and $f^{*}$ for $V h o m^{r}$, Vhom and $H_{o m}$. The proof of the following result is similar to the proof of Proposition 5.4. 
Proposition 5.12. (a) The maps $f_{*}$ and $f^{*}$ are $V$-homomorphisms.

(b) $\operatorname{Vhom}^{r}(N, \square)$ is a covariant functor from $\operatorname{Mod}_{V}$ to $\operatorname{Mod}_{V}$.

(c) $\operatorname{Vhom}^{r}(\square, N)$ is a contravariant functor from $\operatorname{Mod}_{V}$ to $\operatorname{Mod}_{V}$.

Now, we shall present some properties of $V_{h o m}$.

Proposition 5.13. Let $M$ be a $V$-module. Then $\operatorname{Vhom}^{r}(V, M)$ is isomorphic to $M$ as a $V$ module. The isomorphism is given by $\Psi_{M}: \operatorname{Vhom}^{r}(V, M) \rightarrow M$ with $\Psi_{M}(f)=\operatorname{Res}_{z} z^{-1} f_{z}(\mathbf{1})$. Moreover, if $g \in \operatorname{Hom}_{V}(M, N)$, then the following diagram commutes:

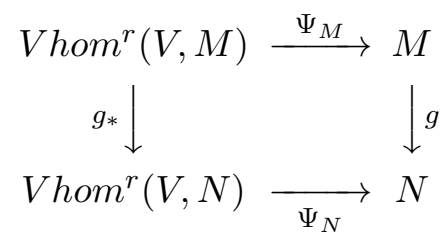

proving that $\Psi$ is a natural isomorphism from $\operatorname{Vhom}^{r}(V, \square)$ to the identity functor on $M o d_{V}$.

Proof. We define a map

$$
\Phi: M \longrightarrow \operatorname{Vhom}(V, M)
$$

given by $[\Phi(u)]_{z}(a)=a_{\dot{z}} u$, for $u \in M$. We should check that $[\Phi(u)]_{z} \in \operatorname{Vhom}^{r}(V, M)$. By definition, we have

$$
[\Phi(u)]_{z}(d a)=(d a) \dot{z} u=\frac{d}{d z}\left(a_{\dot{z}} u\right)=\frac{d}{d z}[\Phi(u)]_{z}(a) .
$$

Using associativity, for any $b \in V$, there is $k \in \mathbb{N}$ depending only on $b$ and $u$ (see Remark 2.4) such that

$$
(z+x)^{k} b_{z \dot{+} x}\left(a_{\dot{x}} u\right)=(z+x)^{k}\left(b_{\dot{z}} a\right)_{\dot{x}} u
$$

for all $a \in V$. Thus,

$$
(z+x)^{k} b_{z \dot{+} x}\left([\Phi(u)]_{x}(a)\right)=(z+x)^{k}\left([\Phi(u)]_{x}\left(b_{\dot{z}} a\right)\right)
$$

for all $a \in V$, proving that $[\Phi(u)]_{z} \in V \operatorname{Vhom}^{r}(V, M)$. It is easy to see using the commutator formula for modules (2.12) that the linear map $\Phi$ from $M$ to $V h o m^{r}(V, M)$ is a $V$-homomorphism.

Conversely, given any $f_{z} \in \operatorname{Vhom}^{r}(V, M)$, we will prove that $f=\Phi\left(\Psi_{M}(f)\right)$. If we write $f_{z}=\sum_{n \in \mathbb{Z}} f_{n} z^{-n-1}$, then by definition $f_{n}(d a)=n f_{n-1}(a)$. In particular, $n f_{n-1}(\mathbf{1})=0$ for all $n \in \mathbb{Z}$. Therefore, $u:=f_{-1}(\mathbf{1})=f_{z}(\mathbf{1}) \in M$. Now, for any $a \in V$ there exists $k \in \mathbb{N}$ such that $(z+x)^{k} f_{z}\left(a_{\dot{x}} b\right)=(z+x)^{k} a_{x \dot{+} z}\left(f_{z}(b)\right)$ for all $b \in V$. Then

$$
\begin{aligned}
z^{k}\left(a_{\dot{z}} u\right) & \left.=(x+z)^{k} a_{x \dot{+} z}\left(f_{z}(\mathbf{1})\right)_{\left.\right|_{x=0}}=(x+z)^{k} f_{z}\left(a_{\dot{x}} \mathbf{1}\right)\right)_{\left.\right|_{x=0}}=(x+z)^{k} f_{z}\left(e^{x d} a\right)_{\left.\right|_{x=0}} \\
& =(x+z)^{k} e^{x \frac{d}{d z}} f_{z}(a)_{\left.\right|_{x=0}}=(x+z)^{k} f_{x+z}(a)=z^{k} f_{z}(a) .
\end{aligned}
$$

Thus, $f=\Phi\left(\Psi_{M}(f)\right)$. Therefore $\operatorname{Vhom}^{r}(V, M)$ is isomorphic to $M$ as a $V$-module. Finally, we have

$$
\Psi_{N}\left(g_{*}(\psi)\right)=\operatorname{Res}_{z} z^{-1}\left[g_{*}(\psi)\right]_{z}(\mathbf{1})=\operatorname{Res}_{z} z^{-1} g\left(\psi_{z}(\mathbf{1})\right)=g\left(\Psi_{M}(\psi)\right),
$$

finishing the proof.

The following result could be thought as a motivation for the definition of $V h o m^{r}$ and the action of $V$ on it. The proof is similar to the proof of Theorem 5.6 
Theorem 5.14. (Adjoint isomorphism, second version) Let $M, N$ and $W$ be $V$-modules. Then there is a natural isomorphism of $\mathbf{k}$-vector spaces

$$
\tau_{M, N, W}^{\prime}: \operatorname{Hom}_{V}(N \underset{V}{\otimes} M, W) \longrightarrow \operatorname{Hom}_{V}\left(M, \operatorname{Vhom}^{r}(N, W)\right)
$$

sending $f: N \underset{V}{\otimes} M \rightarrow W$ to

$$
\left[\tau_{M, N, W}^{\prime}(f)\right](u): v \mapsto f\left(v \underset{z}{\otimes_{2}} u\right)
$$

for $u \in M$ and $v \in N$. That is, fixing any two of $M, N, W$, each $\tau_{M, N, W}^{\prime}$ is a natural isomorphism.

If we consider all the results together, we obtain:

Corollary 5.15. For any $V$-modules $M, N$ and $W$, we have the following linear isomorphisms

$$
\begin{aligned}
\left(\begin{array}{c}
W \\
M, N
\end{array}\right) & \simeq V \text {-Bilinear }(M, N ; W) \simeq \operatorname{Hom}_{V}(M \underset{V}{\otimes} N, W) \\
& \simeq \operatorname{Hom}_{V}(M, \operatorname{Vhom}(N, W)) \simeq \operatorname{Hom}_{V}\left(N, \operatorname{Vhom}^{r}(M, W)\right) .
\end{aligned}
$$

\section{Properties of the tensor product}

We start this section by proving the commutativity of the tensor product. Then we prove the associativity of the tensor product under certain (algebraic and natural) necessary and sufficient conditions.

The transpose of a vertex bilinear map (or an intertwining operator) $F_{z}$ is defined as

$$
\left(F_{z}\right)^{t}(u, v):=e^{z d} F_{-z}(v, u)
$$

and it is a vertex bilinear map (or an intertwining operator) of the corresponding type. We have the following result (cf. Proposition 5.1.6, Proposition 5.1.7 and Theorem 6.2.3 in [Li1]):

Theorem 6.1. Let $M$ and $N$ be $V$-modules.

(a) There is a natural $V$-isomorphism

$$
\varphi_{M}: V \underset{V}{\otimes} M \longrightarrow M
$$

where $\varphi_{M}(a \underset{z}{\otimes} u)=a_{\dot{z}}^{M} u$ for $a \in V$ and $u \in M$, and $(M \underset{\dot{z}}{M})$ is a tensor product of $(V, M)$. Symmetrically, $\left(M,\left(\begin{array}{c}M \\ \dot{z}\end{array}\right)^{t}\right)$ is a tensor product of $(M, V)$.

(b) If $\left(M \otimes_{V} N, \underset{z}{\otimes}\right)$ is a tensor product of the pair $(M, N)$, then $\left(M \underset{V}{\otimes} N,(\underset{z}{\otimes})^{t}\right)$ is a tensor product of the pair $(N, M)$.

(c) The map

$$
\begin{aligned}
\tau: M{\underset{V}{\otimes}}_{\otimes} N & \longrightarrow N \underset{V}{\otimes} M \\
u \otimes_{z} v & \longmapsto e^{z d}\left(v{\underset{-z}{\otimes}}^{\otimes} u\right)
\end{aligned}
$$

is a natural $V$-isomorphism in the sense that the following diagram commutes 


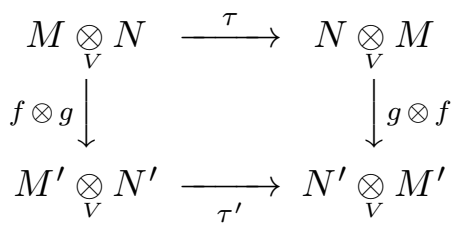

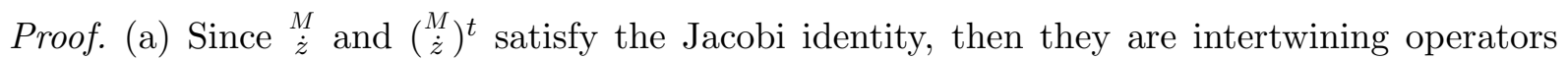
of the corresponding type. Let $W$ be any $V$-module, and let $I_{z}$ be an intertwining operator of type $\left(\begin{array}{c}W \\ V, M\end{array}\right)$. Since $\frac{d}{d z} I_{z}(\mathbf{1}, u)=I_{z}(d \mathbf{1}, u)=0$, then $I_{z}(\mathbf{1}, u)$ is independent of $z$. Using the commutator formula (2.19), we obtain that $I_{z}(\mathbf{1}, \cdot)$ commutes with any operator $a_{\dot{x}}$ for $a \in V$, namely

$$
a_{\dot{x}}^{W} I_{z}(\mathbf{1}, u)=I_{z}\left(\mathbf{1}, a_{\dot{x}}^{M} u\right) .
$$

Then, $\psi(u):=I_{z}(\mathbf{1}, u)$ is a $V$-homomorphism from $M$ to $W$ and it satisfies the universal property if $I_{z}(a, u)=\psi\left(a_{\dot{z}}^{M} u\right)=I_{z}\left(\mathbf{1}, a_{\dot{z}}^{M} u\right)$. But, using the iterated formula (2.18) and (6.2), we have

$$
\begin{aligned}
I_{z}(a, u) & =\operatorname{Res}_{x} x^{-1} I_{z}\left(a_{\dot{x}} \mathbf{1}, u\right) \\
& =\operatorname{Res}_{x} \operatorname{Res}_{y} x^{-1}\left[\delta(y-z, x) a_{\dot{y}}^{W} I_{z}(\mathbf{1}, u)-\delta(-z+y, x) I_{z}\left(\mathbf{1}, a_{\dot{y}}^{M} u\right)\right] \\
& =\operatorname{Res}_{y}\left[(y-z)^{-1}-(-z+y)^{-1}\right] I_{z}\left(\mathbf{1}, a_{\dot{y}}^{M} u\right)=\operatorname{Res}_{y} \delta(y, z) I_{z}\left(\mathbf{1}, a_{\dot{y}}^{M} u\right)=I_{z}\left(\mathbf{1}, a_{\dot{z}}^{M} u\right)
\end{aligned}
$$

for $a \in V, u \in M$, proving that $(M, \underset{\dot{z}}{M})$ is a tensor product of $(V, M)$. Let $F_{z}: V \times M \rightarrow M((z))$ be the $V$-bilinear map defined by $F_{z}(a, u)=a_{\dot{z}}^{M} u$. Then, it induces a $V$-homomorphism $\varphi_{M}$ : $V \otimes M \longrightarrow M$ with $\varphi_{M}(a \underset{z}{\otimes} u)=a_{\dot{z}}^{M} u$. By the uniqueness of the tensor product, $\varphi_{M}$ is a $V$-isomorphism. Naturality is proved by showing commutativity of the following diagram for $f \in \operatorname{Hom}_{V}(M, N)$

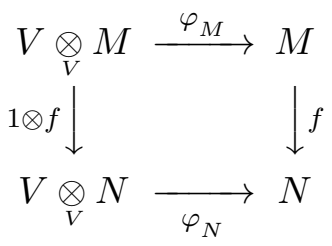

It suffices to check the maps on generators:

$$
f\left(\varphi_{M}\left(a \otimes_{z} u\right)\right)=f\left(a_{\dot{z}} u\right)=a_{\dot{z}} f(u)=\varphi_{N}\left(a \otimes_{z} f(u)\right)=\varphi_{N}\left((1 \otimes f)\left(a \otimes_{z} u\right)\right) .
$$

Similarly, the universal property can be proved for $\left(M,\left(\begin{array}{c}M \\ \dot{z}\end{array}\right)^{t}\right)$, or it follows by using (b).

(b) Let $W$ be any $V$-module and let $I_{z} \in V$ - $\operatorname{Bilinear}(N, M ; W)$. It is easy to see that there is a $V$-homomorphism $\psi$ from $M \underset{V}{\otimes} N$ to $W$ such that $\left(I_{z}\right)^{t}=\psi \circ \underset{z}{\otimes}$ if and only if $I_{z}=\psi \circ(\underset{z}{\otimes})^{t}$, finishing (b).

(c) Let $F_{z}: M \times N \rightarrow(N \underset{V}{\otimes} M)((z))$ be given by $F_{z}(u, v)=e^{z d}(v \underset{-z}{\otimes} u)$ for $u \in M$ and $v \in N$. It easy to see that $F_{z}$ is a vertex bilinear map, and so there is a unique $V$-homomorphism $\tau: M \underset{V}{\otimes} N \rightarrow N \underset{V}{\otimes} M$ with $\tau(u \underset{z}{\otimes} v)=e^{z d}\left(v{\underset{-z}{z}}_{\otimes} u\right)$. Similarly, interchanging the roles of $M$ and $N$, we obtain a $V$-homomorphism $\tilde{\tau}: N \underset{V}{\otimes} M \rightarrow M \underset{V}{\otimes} N$ with $\tilde{\tau}(v \underset{z}{\otimes} u)=e^{z d}\left(u \otimes_{-z}^{\otimes} v\right)$. Both 
composite of these maps are obviously identity maps, and so $\tau$ is a $V$-isomorphism. The proof of naturality is the following:

$$
\tau^{\prime}\left((f \otimes g)\left(u \otimes_{z} v\right)\right)=\tau^{\prime}(f(u) \underset{z}{\otimes} g(v))=e^{z d}(g(v) \underset{-z}{\otimes} f(u))=(g \otimes f)(\tau(u \underset{z}{\otimes} v)) .
$$

Now, we prove the associativity of the tensor product under certain (algebraic and natural) necessary and sufficient condition. We shall use the universal property of the tensor product instead of the explicit construction, following in part the ideas in [DLM] but using algebraic methods instead of analytic ones (by the way, [DLM] is full of typos that can be easily fixed). We found a very natural condition that simplify Huang's convergence assumptions.

Let $M, N$ and $W$ be three $V$-modules. We assume for the rest of this section the existence of the following tensor products $M \underset{V}{\otimes} N,(M \underset{V}{\otimes} N) \underset{V}{\otimes} W, N \underset{V}{\otimes} W$, and $M \underset{V}{\otimes}(N \underset{V}{\otimes} W)$.

The next two propositions immediately follow from the universal property of tensor product in Definition 4.4.

Proposition 6.2. The tensor products $N \otimes_{V} W$ and $M \otimes_{V}(N \underset{V}{\otimes} W)$ (and the corresponding vertex bilinear maps) satisfy the following universal property: For any $V$-modules $Y$ and $Z$, and any vertex bilinear maps $H \in V$-Bilinear $(N, W ; Z)$ and $I \in V$-Bilinear $(M, Z ; Y)$, there exists a unique $V$-homomorphism $g: M \underset{V}{\otimes}(N \underset{V}{\otimes} W) \rightarrow Y$ such that

$$
g\left(u \otimes_{z}\left(v \otimes_{y} w\right)\right)=I_{z}\left(u, H_{y}(v, w)\right)
$$

for all $u \in M, v \in N$ and $w \in W$. This universal property characterizes $M \underset{V}{\otimes}(N \underset{V}{\otimes} W)$ uniquely.

Similarly, we have

Proposition 6.3. The tensor products $M \underset{V}{\otimes} N$ and $(M \underset{V}{\otimes} N) \underset{V}{\otimes} W$ (and the corresponding vertex bilinear maps) satisfy the following universal property: For any $V$-modules $Y$ and $Z$, and any vertex bilinear maps $F \in V$-Bilinear $(M, N ; Z)$ and $G \in V$-Bilinear $(Z, W ; Y)$, there exists a unique $V$-homomorphism $f:(M \underset{V}{\otimes} N) \underset{V}{\otimes} W \rightarrow Y$ such that

$$
f((u \underset{z}{\otimes} v) \underset{y}{\otimes} w)=G_{y}\left(F_{z}(u, v), w\right)
$$

for all $u \in M, v \in N$ and $w \in W$. This universal property characterizes $(M \underset{V}{\otimes} N) \underset{V}{\otimes} W$ uniquely.

Now, we can state the main result of this section.

Theorem 6.4. Let $M, N$ and $W$ be three $V$-modules. Assume the existence of the following tensor products $M \underset{V}{\otimes} N,(M \underset{V}{\otimes} N) \underset{V}{\otimes} W, N \underset{V}{\otimes} W$, and $M \underset{V}{\otimes}(N \underset{V}{\otimes} W)$. Then: there exists a unique $V$ - isomorphism

such that (coefficient-wise)

$$
f:(M \underset{V}{\otimes} N) \underset{V}{\otimes} W \rightarrow M \underset{V}{\otimes}(N \underset{V}{\otimes} W)
$$

$$
f((u \underset{x}{\otimes} v) \underset{y}{\otimes} w)=u \underset{x+y}{\otimes}(v \underset{y}{\otimes} w)
$$

for all $u \in M, v \in N$ and $w \in W$ if and only if the following expressions exist and satisfy:

(1) for any $u \in M, v \in N$ and $w \in W$

$$
u \underset{x+y}{\otimes}(v \underset{y}{\otimes} w) \in[M \underset{V}{\otimes}(N \underset{V}{\otimes} W)]((y))((x))
$$

where the number of negative powers of $x$ depends only on $u$ and $v$, and 
(2) for any $u \in M, v \in N$ and $w \in W$

$$
(u \underset{x-y}{\otimes} v) \underset{y}{\otimes} w \in[(M \underset{V}{\otimes} N) \underset{V}{\otimes} W]((x))((y))
$$

where the number of negative powers of $y$ depends only on $v$ and $w$.

Observe that the expressions always exist, except for $(u \underset{x-y}{\otimes} v) \underset{y}{\otimes} w$. We also have that

$$
(u \underset{x}{\otimes} v) \underset{y}{\otimes} w \in[(M \underset{V}{\otimes} N) \underset{V}{\otimes} W]((y))((x)),
$$

where the finite number of negative powers in $x$ depends only on $u \in M$ and $v \in N$, and

$$
u \underset{x}{\otimes}(v \underset{y}{\otimes} w) \in[M \underset{V}{\otimes}(N \underset{V}{\otimes} W)]((x))((y)),
$$

where the finite number of negative powers in $y$ depends only on $v \in N$ and $w \in W$.

A simple argument that prove the "only if" part of the theorem, is the following: suppose that there exists the $V$-isomorphism $f$. Then, by considering the left hand side of $f((u \underset{x}{\otimes} v) \underset{y}{\otimes} w)=$ $u \otimes\left(v \otimes \otimes_{y} w\right)$, and using (6.5), we have that the right hand side must satisfy (6.3). Since $f$ is an isomorphism, then it is clear that $g: M \otimes_{V}(N \underset{V}{\otimes} W) \rightarrow\left(M \otimes_{V}^{\otimes} N\right) \underset{V}{\otimes} W$ with $g\left(u \otimes_{x}\left(v \otimes_{y} w\right)\right)=\left(u \underset{x-y}{\otimes} v \otimes_{y} w\right.$ is well defined and it is the inverse of $f$, and using (6.6), we have that (6.4) must be satisfied.

By using the vertex bilinear properties (that are preserved by homomorphism), one can see why we should send $(u \underset{x}{\otimes} v) \underset{y}{\otimes} w$ to $u \underset{x+y}{\otimes}(v \underset{y}{\otimes} w)$ instead of $u \underset{x}{\otimes}(v \underset{y}{\otimes} w)$ when we construct the isomorphism.

The rest of this section is devoted to the proof of the "if" part of the associativity theorem. The sketch of proof or the basic idea of the proof is the following: first, we define $F: M \times N \rightarrow$ $\operatorname{Vhom}(W, M \underset{V}{\otimes}(N \underset{V}{\otimes} W))((z))$ given by

$$
\left[F_{z}(u, v)\right]_{y}(w)=u \underset{z+y}{\otimes}(v \underset{y}{\otimes} w)
$$

and we prove that $F$ is a vertex bilinear map of type $\left(M, N ; \operatorname{Vhom}\left(W, M \otimes_{V}\left(N \otimes_{V} W\right)\right)\right)$. Then we take $G$ as the natural vertex bilinear map of type $(\operatorname{Vhom}(W, M \underset{V}{\otimes}(N \underset{V}{\otimes} W)), W ; M \underset{V}{\otimes}(N \underset{V}{\otimes} W))$ given by $G_{y}(q, w)=q_{y}(w)$ for $q \in \operatorname{Vhom}(W, M \underset{V}{\otimes}(N \underset{V}{\otimes} W))$ and $w \in W$. After that, we apply Proposition 6.3 to the vertex bilinear maps $F$ and $G$ to get a $V$-homomorphism $f$ : $(M \underset{V}{\otimes} N) \underset{V}{\otimes} W \rightarrow M \underset{V}{\otimes}(N \underset{V}{\otimes} W)$ such that $f((u \underset{z}{\otimes} v) \underset{y}{\otimes} w)=u \underset{z+y}{\otimes}(v \underset{y}{\otimes} w)$. Similarly, we define $H: N \times W \rightarrow \operatorname{Vhom}^{r}(M,(M \underset{V}{\otimes} N) \underset{V}{\otimes} W)((y))$ given by

$$
\left[H_{y}(v, w)\right]_{z}(u)=(u \underset{z-y}{\otimes} v) \underset{y}{\otimes} w
$$

and we prove that $H$ is a vertex bilinear map of type $\left(N, W ; \operatorname{Vhom}^{r}\left(M,\left(M \otimes_{V} N\right) \underset{V}{\otimes} W\right)\right)$. Then we take $I$ as the natural vertex bilinear map of type $\left(M, \operatorname{Vhom}^{r}(M,(M \underset{V}{\otimes} N) \underset{V}{\otimes} W) ;(M \underset{V}{\otimes} N) \underset{V}{\otimes} W\right)$ given by $I_{y}(u, h)=h_{y}(u)$ for $h \in \operatorname{Vhom}^{r}(M,(M \underset{V}{\otimes} N) \underset{V}{\otimes} W)$ and $u \in M$. After that, we apply Proposition 6.2 to the vertex bilinear maps $H$ and $I$ to get a $V$-homomorphism $g$ : $M \underset{V}{\otimes}(N \underset{V}{\otimes} W) \rightarrow(M \underset{V}{\otimes} N) \underset{V}{\otimes} W$ such that $g(u \underset{z}{\otimes}(v \underset{y}{\otimes} w))=(u \underset{z-y}{\otimes} v) \underset{y}{\otimes} w$, obtaining the desired isomorphisms $f$ and $g$. This is the algebraic and non-graded version of the proof in [DLM]. 
For the rest of this section we assume that all the tensor products exist, and we assume the conditions (6.3)-(6.4) about the Laurent series in the statement of the theorem.

For any fixed $u \in M$ and $v \in N$, we define a linear map

$$
\psi(u, v ; z, y): W \rightarrow[(M \underset{V}{\otimes} N) \underset{V}{\otimes} W]((y))((z))
$$

as

$$
\psi(u, v ; z, y)(w):=u \underset{z+y}{\otimes}(v \underset{y}{\otimes} w)
$$

for any $w \in W$. Using (6.3), we have

$$
\psi(u, v ; z, y)=\sum_{n \in \mathbb{Z}} \psi_{n}(u, v ; y) z^{-n-1}
$$

with $\psi_{k}(u, v ; y) \equiv 0$ for $k$ sufficiently large and

$$
\psi_{n}(u, v ; y): W \rightarrow[M \underset{V}{\otimes}(N \underset{V}{\otimes} W)]((y)) .
$$

Proposition 6.5. For any $u \in M, v \in N$ and $n \in \mathbb{Z}$, we have

$$
\psi_{n}(u, v ; y) \in \operatorname{Vhom}(W, M \underset{V}{\otimes}(N \underset{V}{\otimes} W)) .
$$

Proof. For $u \in M, v \in N, w \in W$ and $n \in \mathbb{Z}$, we have

$$
\begin{aligned}
\psi(u, v ; z, y)(d w) & =u \underset{z+y}{\otimes}(v \underset{y}{\otimes} d w)=u \underset{z+y}{\otimes}\left(d-\frac{d}{d y}\right)(v \underset{y}{\otimes} w)=\left(d-\frac{d}{d y}\right)(u \underset{z+y}{\otimes}(v \underset{y}{\otimes} w)) \\
& =\left(d-\frac{d}{d y}\right) \psi(u, v ; z, y)(w) .
\end{aligned}
$$

Then $\psi_{n}(u, v ; y)$ satisfies $(a)$ in Definition 5.1. Since $\psi_{m}(u, v ; y)=0$ for all $m \geq N$, for some $N \in \mathbb{N}$, then

$$
\operatorname{Res}_{z} z^{m} f(y, z) \psi(u, v ; z, y)=0
$$

for any $f \in \mathbf{k}[y, z]$. Now, using the Definition 4.1.(3) where the exponents depend on certain variables, we have that for any $a \in V$ and $n \in \mathbb{Z}$, we take $k \in \mathbb{N}$ such that $k+n \geq N$ and

$$
\begin{aligned}
(x-z)^{k} a_{\dot{x}}(u \underset{z}{\otimes}(v \underset{y}{\otimes} w)) & =(x-z)^{k} u \underset{z}{\otimes}\left(a_{\dot{x}}(v \underset{y}{\otimes} w)\right) & & \text { for all } v \in N, w \in W, \\
(x-y)^{k} a_{\dot{x}}(v \underset{y}{\otimes} w) & =(x-y)^{k}\left(v \underset{y}{\otimes} a_{\dot{x}} w\right) & & \text { for all } w \in W .
\end{aligned}
$$

Then, using (6.7), (6.8) and (6.9), we have 


$$
\begin{aligned}
(x-y)^{3 k} & a_{\dot{x}} \psi_{n}(u, v ; y)(w)=\operatorname{Res}_{z} z^{n}(x-y)^{3 k} a_{\dot{x}} \psi(u, v ; z, y)(w) \\
& =\sum_{i=0}^{2 k}\left(\begin{array}{c}
2 k \\
i
\end{array}\right) \operatorname{Res}_{z}(x-z-y)^{i} z^{n+2 k-i}(x-y)^{k} a_{\dot{x}} \psi(u, v ; z, y)(w) \\
& =\sum_{i=k}^{2 k}\left(\begin{array}{c}
2 k \\
i
\end{array}\right) \operatorname{Res}_{z}(x-z-y)^{i} z^{n+2 k-i}(x-y)^{k} a_{\dot{x}}(u \underset{z+y}{\otimes}(v \underset{y}{\otimes} w)) \\
& =\sum_{i=k}^{2 k}\left(\begin{array}{c}
2 k \\
i
\end{array}\right) \operatorname{Res}_{z}(x-z-y)^{i} z^{n+2 k-i}(x-y)^{k} \underset{z+y}{\left.\underset{y}{\otimes}\left(v \underset{y}{\otimes} a_{\dot{x}} w\right)\right)} \\
& =\sum_{i=k}^{2 k}\left(\begin{array}{c}
2 k \\
i
\end{array}\right) \operatorname{Res}_{z}(x-z-y)^{i} z^{n+2 k-i}(x-y)^{k} \psi(u, v ; z, y)\left(a_{\dot{x}} w\right) \\
& =\sum_{i=0}^{2 k}\left(\begin{array}{c}
2 k \\
i
\end{array}\right) \operatorname{Res}_{z}(x-z-y)^{i} z^{n+2 k-i}(x-y)^{k} \psi(u, v ; z, y)\left(a_{\dot{x}} w\right) \\
& =\operatorname{Res}_{z} z^{n}(x-y)^{3 k} \psi(u, v ; z, y)\left(a_{\dot{x}} w\right) \\
& =(x-y)^{3 k} \psi_{n}(u, v ; y)\left(a_{\dot{x}} w\right) \quad \text { for all } w \in W .
\end{aligned}
$$

Thus $\psi_{n}(u, v ; y) \in \operatorname{Vhom}(W, M \underset{V}{\otimes}(N \underset{V}{\otimes} W))$ for any $n \in \mathbb{Z}, u \in M, v \in N$.

Now, using the previous proposition, we define the $\mathbf{k}$-bilinear map

$$
F: M \times N \rightarrow \operatorname{Vhom}(W, M \underset{V}{\otimes}(N \underset{V}{\otimes} W))((z))
$$

given by

$$
\left[F_{z}(u, v)\right]_{y}(w)=\psi(u, v ; z, y)(w)=u \underset{z+y}{\otimes}(v \underset{y}{\otimes} w)=\sum_{n \in \mathbb{Z}} \psi_{n}(u, v ; y)(w) z^{-n-1},
$$

for any $u \in M, v \in N$ and $w \in W$.

Proposition 6.6. $F_{z}$ is a vertex bilinear map of type $(M, N ; \operatorname{Vhom}(W, M \underset{V}{\otimes}(N \underset{V}{\otimes} W)))$.

Proof. For $u \in M, v \in V$ and $w \in W$, we have

$$
\begin{aligned}
{\left[F_{z}(d u, v)\right]_{y}(w) } & =(d u) \underset{z+y}{\otimes}(v \underset{y}{\otimes} w)=\frac{d}{d x}(u \underset{x}{\otimes}(v \underset{y}{\otimes} w))_{\mid x=z+y}=\frac{d}{d z}(u \underset{z+y}{\otimes}(v \underset{y}{\otimes} w)) \\
& =\left[\frac{d}{d z} F_{z}(u, v)\right]_{y}(w),
\end{aligned}
$$

and

$$
\begin{aligned}
{\left[\left(d-\frac{d}{d z}\right) F_{z}(u, v)\right]_{y}(w) } & =\left(\frac{d}{d y}-\frac{d}{d z}\right)\left(\left[F_{z}(u, v)\right]_{y}(w)\right)=\left(\frac{d}{d y}-\frac{d}{d z}\right)(u \underset{z+y}{\otimes}(v \underset{y}{\otimes} w))= \\
& =u \underset{z+y}{\otimes} \frac{d}{d y}(v \underset{y}{\otimes} w)=u \underset{z+y}{\otimes}(d v \underset{y}{\otimes} w)=\left[F_{z}(u, d v)\right]_{y}(w) .
\end{aligned}
$$

Then $F_{z}$ satisfies the properties with respect to $d$. Now, we prove the commutator formula, from which we obtain (4.2). For $a \in V, u \in M, v \in N$ and $w \in W$, using the action on Vhom, we 
have

$$
\begin{aligned}
{\left[a_{\dot{x}} F_{z}(u, v)\right]_{y}(w) } & =a_{x \dot{+} y}\left(\left[F_{z}(u, v)\right]_{y}(w)\right)-\left[F_{z}(u, v)\right]_{y}\left(a_{x \dot{+} y} w-a_{y \dot{+} x} w\right) \\
& =a_{x \dot{y} y}\left(u \underset{z+y}{\otimes}\left(v_{\dot{y}} w\right)\right)-u \underset{z+y}{\otimes}\left(v \underset{y}{\otimes}\left(a_{x \dot{+} y} w-a_{y \dot{+} x} w\right)\right)
\end{aligned}
$$

and

$$
\left[F_{z}\left(u, a_{\dot{x}} v\right)\right]_{y}(w)=u \underset{z+y}{\otimes}\left(a_{\dot{x}} v \underset{y}{\otimes} w\right) .
$$

Then, using associator formula (2.17) and the action on the tensor product, we have

$$
\begin{aligned}
{\left[a_{\dot{x}} F_{z}(u, v)\right]_{y}(w) } & -\left[F_{z}\left(u, a_{\dot{x}} v\right)\right]_{y}(w)= \\
& =a_{x \dot{+} y}(u \underset{z+y}{\otimes}(v \underset{y}{\otimes} w))-u \underset{z+y}{\otimes}\left(a_{\dot{x}} v \underset{y}{\otimes} w+v \underset{y}{\otimes}\left(a_{x \dot{+} y} w-a_{y \dot{x}} w\right)\right) \\
& =a_{x \dot{+} y}(u \underset{z+y}{\otimes}(v \underset{y}{\otimes} w))-u \underset{z+y}{\otimes} a_{x \dot{+} y}(v \underset{y}{\otimes} w) \\
& =\left(a_{x-z} u-a_{-z+x} u\right) \underset{z+y}{\otimes}(v \underset{y}{\otimes} w) \\
& =\left[F_{z}\left(a_{x-z} u-a_{-\dot{z}+x} u, v\right)\right]_{y}(w)
\end{aligned}
$$

and this gives rise to the commutator formula (2.19) which implies locality or (4.2). Next, we prove associativity or (4.1). For $a \in V$ and $v \in N$, we take $k \in \mathbb{N}$ (depending on $a$ and $v$ ) such that

$$
(x-y)^{k} a_{\dot{x}}(v \underset{y}{\otimes} w)=(x-y)^{k} v \underset{y}{\otimes} a_{\dot{x}} w, \quad \text { for all } w \in W .
$$

Now, using the definition of the action on Vhom, the associator formula (2.17) and (6.10), we have

$$
\begin{aligned}
(x+z)^{k}\left(\left[F_{z}\left(a_{\dot{x}} u, v\right)\right]_{y}(w)-\left[a_{x \dot{y}} F_{z}(u, v)\right]_{y}(w)\right)= \\
=(x+z)^{k}\left(\left(a_{\dot{x}} u\right) \underset{z+y}{\otimes}(v \underset{y}{\otimes} w)-a_{x+\dot{z}+y}(u \underset{z+y}{\otimes}(v \underset{y}{\otimes} w))+\operatorname{Res}_{t} \delta(-y+t, x+z) u \underset{z+y}{\otimes}\left(v \underset{y}{\otimes} a_{\dot{t}} w\right)\right) \\
=(x+z)^{k}\left(\operatorname{Res}_{t}\left[\delta(-y+t, x+z) u \underset{z+y}{\otimes}\left(v \underset{y}{\otimes} a_{\dot{t}} w\right)-\delta(-z-y+t, x) u \underset{z+y}{\otimes} a_{\dot{t}}(v \underset{y}{\otimes} w)\right]\right) \\
=\operatorname{Res}_{t}\left[(x+z)^{k} \delta(-y+t, x+z) \underset{z+y}{\otimes}\left(v \underset{y}{\otimes} a_{\dot{t}} w\right)\right] \\
\quad-\operatorname{Res}_{t}\left[(\delta(x+z,-y+t)-\delta(z+x,-y+t))(-y+t)^{k} u \underset{z+y}{\otimes} a_{\dot{t}}\left(v{\underset{y}{\otimes}}_{y} w\right)\right] \\
=(x+z)^{k} \operatorname{Res}_{t}\left[\delta(z+x,-y+t) u \underset{z+y}{\otimes}\left(v \underset{y}{\otimes} a_{\dot{t}} w\right)\right] \\
=(x+z)^{k} \operatorname{Res}_{t}[\delta(z+x+y, t)-\delta(y+z+x, t)] u \underset{z+y}{\otimes}\left(v \underset{y}{\otimes} a_{\dot{t}} w\right) .
\end{aligned}
$$

Therefore, if we take $l\left(=N_{a, w}\right)$ such that $x^{l} a_{\dot{x}} w \in W[[x]]$, then

$$
(x+z)^{k}(y+z+x)^{l}\left[F_{z}\left(a_{\dot{x}} u, v\right)\right]_{y}(w)=(x+z)^{k}(y+z+x)^{l}\left[a_{x \dot{+} z} F_{z}(u, v)\right]_{y}(w) .
$$

Notice that $(y+x+z)^{-l}=(y+z+x)^{-l}$ and that we are allowed to multiply the right-hand side of (6.11) by $(y+x+z)^{-l}$ and to multiply the left-hand side by $(y+z+x)^{-l}$. Then multiplying both sides by $(y+x+z)^{-l}$ we obtain

$$
(x+z)^{k}\left[F_{z}\left(a_{\dot{x}} u, v\right)\right]_{y}(w)=(x+z)^{k}\left[a_{x \dot{+} z} F_{z}(u, v)\right]_{y}(w) .
$$

Since $k$ does not depend on $w$, we immediately have (4.1), as desired (cf. this argument with the last part of the proof of Theorem 5.22 in [Li3]). 
Using Proposition 6.6, we have $F \in V$ - $\operatorname{Bilinear}(M, N ; \operatorname{Vhom}(W, M \underset{V}{\otimes}(N \underset{V}{\otimes} W)))$ given by $\left[F_{z}(u, v)\right]_{y}(w)=u \underset{z+y}{\otimes}(v \underset{y}{\otimes} w)$, and the natural vertex bilinear map

$$
G \in V \text { - Bilinear }(\operatorname{Vhom}(W, M \underset{V}{\otimes}(N \underset{V}{\otimes} W)), W ; M \underset{V}{\otimes}(N \underset{V}{\otimes} W))
$$

given by $G_{y}(q, w)=q_{y}(w)$. Now, applying Proposition 6.3 to these vertex bilinear maps, we obtain:

Proposition 6.7. There exists a unique $V$-homomorphism $f:(M \underset{V}{\otimes} N) \underset{V}{\otimes} W \rightarrow M \underset{V}{\otimes}(N \underset{V}{\otimes} W)$ such that

$$
f((u \underset{z}{\otimes} v) \underset{y}{\otimes} w)=u \underset{z+y}{\otimes}(v \underset{y}{\otimes} w)
$$

for $u \in M, v \in N$ and $w \in W$.

For any $v \in N$ and $w \in W$, we define a linear map

$$
\phi(v, w ; z, y): M \rightarrow[(M \underset{V}{\otimes} N) \underset{V}{\otimes} W]((z))((y))
$$

given by

$$
\phi(v, w ; z, y)(u):=(u \underset{z-y}{\otimes} v) \underset{y}{\otimes} w .
$$

It follows from the assumption (6.4), that this expression exists and if we take

$$
\phi(v, w ; z, y)=\sum_{n \in \mathbb{Z}} \phi_{n}(v, w ; z) y^{-n-1}
$$

then $\phi_{k}(v, w ; z) \equiv 0$ for $k$ sufficiently large and

$$
\phi_{n}(v, w ; z): M \rightarrow[(M \underset{V}{\otimes} N) \underset{V}{\otimes} W]((z)) .
$$

Proposition 6.8. For any $v \in N, w \in W$ and $n \in \mathbb{Z}$, we have

$$
\phi_{n}(v, w ; z) \in \operatorname{Vhom}^{r}(M,(M \underset{V}{\otimes} N) \underset{V}{\otimes} W) .
$$

Proof. For $v \in N, w \in W$ and $n \in \mathbb{Z}$, we have

$$
\begin{aligned}
& \phi(v, w ; z, y)(d u)=(d u \underset{z-y}{\otimes} v) \underset{y}{\otimes} w=\left[\frac{d}{d x}((u \underset{x}{\otimes} v) \underset{y}{\otimes} w)\right]_{x=z-y}=\frac{d}{d z}((u \underset{z-y}{\otimes} v) \underset{y}{\otimes} w) \\
& =\frac{d}{d z} \phi(v, w ; z, y)(u) .
\end{aligned}
$$

Then, $\phi_{n}(v, w ; z)$ satisfies (a) in the definition of $V h o m^{r}$. Let $N \in \mathbb{N}$ be such that $\phi_{m}(v, w ; z)=$ 0 for all $m \geq N$. Now, for any $a \in V$ and $n \in \mathbb{Z}$, we take $k \in \mathbb{N}$ such that $k+n \geq N$, and

$$
\begin{aligned}
& x^{k} a_{\dot{x}}\left(u \otimes_{z} v\right)=x^{k}\left(a_{x-z} u\right) \underset{z}{\otimes} v \quad \text { for all } u \in M, \\
& x^{k} a_{\dot{x}}((u \underset{z}{\otimes} v) \underset{y}{\otimes} w)=x^{k}\left(a_{x \dot{y} y}(u \underset{z}{\otimes} v)\right) \underset{y}{\otimes} w \quad \text { for all } u \in M \text { and } v \in N \text {. }
\end{aligned}
$$


Then, we obtain

$$
\begin{aligned}
& x^{3 k} a_{\dot{x}}\left(\phi_{n}(v, w ; z)(u)\right)=\operatorname{Res}_{y} y^{n} x^{3 k} a_{\dot{x}}(\phi(v, w ; z, y)(u)) \\
& =\operatorname{Res}_{y} \sum_{i=0}^{2 k}\left(\begin{array}{c}
2 k \\
i
\end{array}\right)(x-y)^{2 k-i} y^{n+i} x^{k} a_{\dot{x}}(\phi(v, w ; z, y)(u)) \\
& =\operatorname{Res}_{y} \sum_{i=0}^{k}\left(\begin{array}{c}
2 k \\
i
\end{array}\right)(x-y)^{2 k-i} y^{n+i} x^{k} a_{\dot{x}}(\phi(v, w ; z, y)(u)) \\
& =\operatorname{Res}_{y} \sum_{i=0}^{k}\left(\begin{array}{c}
2 k \\
i
\end{array}\right)(x-y)^{2 k-i} y^{n+i} x^{k}\left(a_{x-y}(u \underset{z-y}{\otimes} v)\right) \underset{y}{\otimes} w \\
& =\operatorname{Res}_{y} \sum_{i=0}^{k}\left(\begin{array}{c}
2 k \\
i
\end{array}\right)(x-y)^{2 k-i} y^{n+i} x^{k}\left(\left(a_{x-z} u\right) \underset{z-y}{\otimes} v\right) \underset{y}{\otimes} w \\
& =\operatorname{Res}_{y} \sum_{i=0}^{2 k}\left(\begin{array}{c}
2 k \\
i
\end{array}\right)(x-y)^{2 k-i} y^{n+i} x^{k}\left(\left(a_{x-z} u\right) \underset{z-y}{\otimes} v\right) \underset{y}{\otimes} w \\
& =\operatorname{Res}_{y} x^{3 k} y^{n}\left(\left(a_{x-z} u\right) \underset{z-y}{\otimes} v\right) \underset{y}{\otimes} w \\
& =\operatorname{Res}_{y} y^{n} x^{3 k}\left(\phi(v, w ; z, y)\left(a_{x-z} u\right)\right) \\
& =x^{3 k} \phi_{n}(v, w ; z)\left(a_{x-z} u\right) \quad \text { for all } u \in M \text {. }
\end{aligned}
$$

Then, $\phi_{n}(v, w ; z) \in \operatorname{Vhom}^{r}(M,(M \underset{V}{\otimes} N) \underset{V}{\otimes} W)$ for all $v \in N, w \in W$ and $n \in \mathbb{Z}$.

Now, we define a k-bilinear map

$$
H_{y}: N \times W \rightarrow \operatorname{Vhom}^{r}(M,(M \underset{V}{\otimes} N) \underset{V}{\otimes} W)((y))
$$

given by

$$
\left[H_{y}(v, w)\right]_{z}(u):=\phi(v, w ; z, y)(u)=(u \underset{z-y}{\otimes} v) \underset{y}{\otimes} w
$$

for any $v \in N, w \in W$ and $u \in M$.

Proposition 6.9. $H_{y}$ is a vertex bilinear map of type $\left(N, W ; \operatorname{Vhom}^{r}(M,(M \underset{V}{\otimes} N) \underset{V}{\otimes} W)\right)$.

Proof. For any $u \in M, v \in N$ and $w \in W$, we have

$$
\begin{aligned}
{\left[H_{y}(d v, w)\right]_{z}(u) } & =(u \underset{z-y}{\otimes} d v) \underset{y}{\otimes} w=\left[\left(d-\frac{d}{d x}\right)(u \underset{x}{\otimes} v)\right]{\underset{y}{y}}_{\left.\right|_{x=z-y}} \\
& =\left(\frac{d}{d y}[(u \underset{x}{\otimes} v) \underset{y}{\otimes} w]\right)_{\mid x=z-y}-\frac{d}{d x}[(u \underset{x}{\otimes} v) \underset{y}{\otimes} w]_{\mid x=z-y} \\
& =\frac{d}{d y}((u \underset{z-y}{\otimes} v) \underset{y}{\otimes} w)=\frac{d}{d y}\left[H_{y}(v, w)\right]_{z}(u),
\end{aligned}
$$

and

$$
\begin{aligned}
{\left[\left(d-\frac{d}{d y}\right) H_{y}(v, w)\right]_{z}(u) } & =\left(d-\frac{d}{d z}-\frac{d}{d y}\right)\left[H_{y}(v, w)\right]_{z}(u)=\left(d-\frac{d}{d z}-\frac{d}{d y}\right)((u \underset{z-y}{\otimes} v) \underset{y}{\otimes} w) \\
& =\left[\left(d-\frac{d}{d y}\right)((u \underset{x}{\otimes} v) \underset{y}{\otimes} w)\right]_{x=z-y}=(u \underset{z-y}{\otimes} v) \underset{y}{\otimes} d w=\left[H_{y}(v, d w)\right]_{z}(u) .
\end{aligned}
$$


Then $H_{y}$ satisfies the properties with respect to $d$. Now, we prove that $H_{y}$ satisfies locality or (4.2). For $a \in V, u \in M$ and $v \in N$, we take $k$ and $n$ positive integers such that

$$
x^{k} a_{\dot{x}} u \in M[[x]] \quad \text { and } \quad x^{n} a_{\dot{x}} v \in N[[x]] .
$$

Given $w \in W$ and using the action on $V h o m^{r}$ and the action on the tensor product, we have

$$
\begin{aligned}
(x-y)^{n}(x-z)^{k} & \left(\left[a_{\dot{x}} H_{y}(v, w)\right]_{z}(u)-\left[H_{y}\left(v, a_{\dot{x}} w\right)\right]_{z}(u)\right)= \\
& =(x-y)^{n}(x-z)^{k}\left(a_{\dot{x}}((u \underset{z-y}{\otimes} v) \underset{y}{\otimes} w)-(u \underset{z-y}{\otimes} v) \underset{y}{\otimes} a_{\dot{x}} w\right) \\
& =(x-y)^{n}(x-z)^{k} \operatorname{Res}_{t} \delta(y+t, x)\left(a_{\dot{t}}(u \underset{z-y}{\otimes} v)\right) \underset{y}{\otimes} w \\
& =(x-y)^{n} \operatorname{Res}_{t} \delta(y+t, x)(t-(z-y))^{k}\left(a_{\dot{t}}(u \underset{z-y}{\otimes} v)\right) \underset{y}{\otimes} w \\
& =(x-y)^{n}(x-z)^{k} \operatorname{Res}_{t} \delta(y+t, x)\left(u \underset{z-y}{\otimes} a_{\dot{t}} v\right) \underset{y}{\otimes} w \\
& =(x-z)^{k} \operatorname{Res}_{t}(\delta(x-y, t)-\delta(-y+x, t))(x-y)^{n}\left(u \underset{z-y}{\otimes} a_{\dot{t}} v\right) \underset{y}{\otimes} w=0
\end{aligned}
$$

Therefore

$$
(x-y)^{n}(x-z)^{k}\left[a_{\dot{x}} H_{y}(v, w)\right]_{z}(u)=(x-y)^{n}(x-z)^{k}\left[H_{y}\left(v, a_{\dot{x}} w\right)\right]_{z}(u) .
$$

Observe that we are allowed to multiply both sides of $(6.12)$ by $(-z+x)^{-k}$, and we obtain

$$
(x-y)^{n}\left[a_{\dot{x}} H_{y}(v, w)\right]_{z}(u)=(x-y)^{n}\left[H_{y}\left(v, a_{\dot{x}} w\right)\right]_{z}(u) .
$$

Since $n$ does not depend on $u$, we have proved locality.

Now, we prove associativity or (4.1). Let $k$ be a positive integer (depending only on $a \in V$ and $w \in W$ ) such that

$$
(x+y)^{k} a_{x \dot{+} y}(q \underset{y}{\otimes} w)=(x+y)^{k}\left(a_{\dot{x}} q\right) \underset{y}{\otimes} w
$$

for any $q \in M \underset{V}{\otimes} N$. Then, using the action on $V h o m^{r},(6.13)$ and the action on the tensor product, we obtain

$$
\begin{aligned}
(x+y)^{k} & {\left[a_{x \dot{+} y} H_{y}(v, w)\right]_{z}(u)=} \\
& =(x+y)^{k} a_{x \dot{y}}((u \underset{z-y}{\otimes} v) \underset{y}{\otimes} w)-\operatorname{Res}_{t} \delta(z+t, x+y)(x+y)^{k}\left(\left(a_{\dot{t}} u\right) \underset{z-y}{\otimes} v\right) \underset{y}{\otimes} w \\
& =(x+y)^{k}\left(a_{\dot{x}}(u \underset{z-y}{\otimes} v)\right) \underset{y}{\otimes} w-\operatorname{Res}_{t} \delta(z+t, x+y)(x+y)^{k}\left(\left(a_{\dot{t}} u\right) \underset{z-y}{\otimes} v\right) \underset{y}{\otimes} w \\
& =(x+y)^{k}\left(u \underset{z-y}{\otimes} a_{\dot{x}} v\right) \underset{y}{\otimes} w+\operatorname{Res}_{t} \delta(x-t, z-y)(x+y)^{k}\left(\left(a_{\dot{t}} u\right) \underset{z-y}{\otimes} v\right) \underset{y}{\otimes} w \\
& =(x+y)^{k}\left(u \operatorname{Res}_{t} \delta(z+t, x+y)(x+y)^{k}\left(\left(a_{\dot{t}} u\right)_{z-y}^{\otimes} v\right) \underset{y}{\otimes} w\right. \\
& =(x+y)^{k}\left[H_{y}\left(a_{\dot{x}} v, w\right)\right]_{z} w \quad \text { for all } u \in M .
\end{aligned}
$$

This proves the associativity or (4.1). Therefore $H$ is a vertex bilinear map.

Using Proposition 6.9, we have $H \in V$ - Bilinear $\left(N, W ; \operatorname{Vhom}^{r}(M,(M \underset{V}{\otimes} N) \underset{V}{\otimes} W)\right)$ given by

$$
\left[H_{y}(u, v)\right]_{z}(u)=(u \underset{z-y}{\otimes} v) \underset{y}{\otimes} w
$$

and the natural vertex bilinear map $I$ of type $\left(M, \operatorname{Vhom}^{r}(M,(M \underset{V}{\otimes} N) \underset{V}{\otimes} W) ;(M \underset{V}{\otimes} N) \underset{V}{\otimes} W\right)$ given by $I_{y}(u, h)=h_{y}(u)$. Now, applying Proposition 6.2 to these vertex bilinear maps, we obtain: 
Proposition 6.10. There exists a unique $V$-homomorphism $g: M \underset{V}{\otimes}(N \underset{V}{\otimes} W) \rightarrow(M \underset{V}{\otimes} N) \underset{V}{\otimes} W$ such that

$$
g(u \underset{z}{\otimes}(v \underset{y}{\otimes} w))=(u \underset{z-y}{\otimes} v) \underset{y}{\otimes} w
$$

for all $u \in M, v \in N$ and $w \in W$.

By Proposition 6.7 and Proposition 6.10, we have:

Theorem 6.11. Let $V$ be a vertex algebra and $M, N$ and $W$ be three $V$-modules. Assume that the tensor products $M \underset{V}{\otimes} N, N \underset{V}{\otimes} W,(M \underset{V}{\otimes} N) \underset{V}{\otimes} W$ and $M \underset{V}{\otimes}(N \underset{V}{\otimes} W)$ exist, and assume that

(1) for any $u \in M, v \in N$ and $w \in W$

$$
u \underset{x+y}{\otimes}(v \underset{y}{\otimes} w) \in[M \underset{V}{\otimes}(N \underset{V}{\otimes} W)]((y))((x))
$$

where the number of negative powers of $x$ depends only on $u$ and $v$, and

(2) for any $u \in M, v \in N$ and $w \in W$

$$
(u \underset{x-y}{\otimes} v) \underset{y}{\otimes} w \in[(M \underset{V}{\otimes} N) \underset{V}{\otimes} W]((x))((y))
$$

where the number of negative powers of $y$ depends only on $v$ and $w$. Then the $V$-homomorphisms $f$ and $g$ in Proposition 6.7 and Proposition 6.10, are $V$-isomorphisms between $M \underset{V}{\otimes}(N \underset{V}{\otimes} W)$ and $(M \underset{V}{\otimes} N) \underset{V}{\otimes} W$.

\section{EXACTNESS OF FUNCTORS}

We will introduce the notions of vertex projective, vertex injective and vertex flat $V$-modules. Roughly speaking, homological algebra is concerned with the question of how much modules differ from being projective, injective and flat.

We do not try to develop homological algebra theory for vertex algebras in this work. This is just an introduction or motivation to the reader to try to develop it from the point of view of a vertex algebra as a generalization of an associative commutative algebra with unit.

Now, we examine the behavior of Vhom with respect to exact sequences.

Theorem 7.1. (Left exactness) (a) If a sequence of $V$-modules

$$
0 \longrightarrow N^{\prime} \stackrel{\varphi}{\longrightarrow} N \stackrel{\psi}{\longrightarrow} N^{\prime \prime}
$$

is exact, then for any $V$-module $M$ we have that

$$
0 \longrightarrow \operatorname{Vhom}\left(M, N^{\prime}\right) \stackrel{\varphi_{*}}{\longrightarrow} \operatorname{Vhom}(M, N) \stackrel{\psi_{*}}{\longrightarrow} \operatorname{Vhom}\left(M, N^{\prime \prime}\right)
$$

is an exact sequence of $V$-modules.

(b) If a sequence of $V$-modules

$$
M^{\prime} \stackrel{\varphi}{\longrightarrow} M \stackrel{\psi}{\longrightarrow} M^{\prime \prime} \longrightarrow 0
$$

is exact, then for any $V$-module $N$ we have that

$$
0 \longrightarrow \operatorname{Vhom}\left(M^{\prime \prime}, N\right) \stackrel{\psi^{*}}{\longrightarrow} \operatorname{Vhom}(M, N) \stackrel{\varphi^{*}}{\longrightarrow} \operatorname{Vhom}\left(M^{\prime}, N\right)
$$

is an exact sequence of $V$-modules. 
Proof. (a) If $0 \longrightarrow N^{\prime} \stackrel{\varphi}{\longrightarrow} N \stackrel{\psi}{\longrightarrow} N^{\prime \prime}$ is exact, then:

(i) $\operatorname{Ker} \varphi_{*}=0$ : if $f \in \operatorname{Ker} \varphi_{*}$ with $f: M \rightarrow N^{\prime}((z))$, then $\varphi_{*}(f)=0$, that is $\varphi\left(f_{z}(u)\right)=0$ for all $u \in M$. Since $\varphi$ is injective, we have $f=0$. Therefore Ker $\varphi_{*}=0$.

(ii) $\operatorname{Im} \varphi_{*} \subseteq \operatorname{Ker} \psi_{*}$ : Since $\operatorname{Im} \varphi=\operatorname{Ker} \psi$ by exactness, we have $\psi \varphi=0$ and hence $\psi_{*} \varphi_{*}=$ $(\psi \varphi)_{*}=0$. Therefore $\operatorname{Im} \varphi_{*} \subseteq \operatorname{Ker} \psi_{*}$.

(iii) $\operatorname{Ker} \psi_{*} \subseteq \operatorname{Im} \varphi_{*}$ : if $g \in \operatorname{Ker} \psi_{*}$, then $\psi g=0$ and $\operatorname{Res}_{z} z^{m} g_{z}(u) \in \operatorname{Ker} \psi=\operatorname{Im} \varphi$ for all $u \in M, m \in \mathbb{Z}$. Since $\varphi$ is a monomorphism, $\varphi: N^{\prime} \rightarrow \operatorname{Im} \varphi$ is an isomorphism. Define $h$ by $h=\varphi^{-1} g$, where $\varphi^{-1}$ is defined on $\operatorname{Im} \varphi$, then $h \in \operatorname{Vhom}\left(M, N^{\prime}\right)$ and $g=\varphi h=\varphi_{*}(h)$. Therefore $\operatorname{Ker} \psi_{*} \subseteq \operatorname{Im} \varphi_{*}$.

(b) If $M^{\prime} \stackrel{\varphi}{\longrightarrow} M \stackrel{\psi}{\longrightarrow} M^{\prime \prime} \longrightarrow 0$ is exact, then:

(i) $\operatorname{Ker} \psi^{*}=0$ : if $f \in \operatorname{Ker} \psi^{*}$ with $f \in \operatorname{Vhom}\left(M^{\prime \prime}, N\right)$, then $\psi^{*}(f)=0$, that is $f_{z}(\psi(u))=0$ for all $u \in M$. Thus, $f_{z}\left(u^{\prime \prime}\right)=0$ for all $u^{\prime \prime} \in \operatorname{Im} \psi$. Since $\psi$ is surjective, we have $f=0$. Therefore $\operatorname{Ker} \psi^{*}=0$.

(ii) $\operatorname{Im} \psi^{*} \subseteq \operatorname{Ker} \varphi^{*}$ : if $g \in \operatorname{Vhom}\left(M^{\prime \prime}, N\right)$, then $\varphi^{*}\left(\psi^{*}(g)\right)=(\psi \varphi)^{*}(g)=0$ because exactness of the original sequence.

(iii) $\operatorname{Ker} \varphi^{*} \subseteq \operatorname{Im} \psi^{*}:$ if $g \in \operatorname{Ker} \varphi^{*}$, then $g: M \rightarrow N((z))$ and $g_{z}\left(\varphi\left(u^{\prime}\right)\right)=0$ for all $u^{\prime} \in M^{\prime}$. If $u^{\prime \prime} \in M^{\prime \prime}$, then $u^{\prime \prime}=\psi(u)$ for some $u \in M$, because $\psi$ is surjective. Define $f: M^{\prime \prime} \rightarrow N((z))$ by $f_{z}\left(u^{\prime \prime}\right)=g_{z}(u)$ if $u^{\prime \prime}=\psi(u)$. Note that $f$ is well defined: if $\psi(u)=\psi(\tilde{u})$, then $u-\tilde{u} \in \operatorname{Ker} \psi=$ $\operatorname{Im} \varphi$, so that $u-\tilde{u}=\varphi\left(u^{\prime}\right)$ for some $u^{\prime} \in M^{\prime}$. Hence, $g_{z}(u)-g_{z}(\tilde{u})=g_{z}(u-\tilde{u})=g_{z}\left(\varphi\left(u^{\prime}\right)\right)=0$ because $g_{z} \varphi=0$. The reader may check that $f$ is a vertex homomorphism. Finally, $\psi^{*}(f)=$ $f \psi=g$, because if $u^{\prime \prime}=\psi(u)$, then $g_{z}(u)=f_{z}\left(u^{\prime \prime}\right)=f(\psi(u))$. Therefore $g \in \operatorname{Im} \psi^{*}$.

We have proved that $\operatorname{Vhom}(M, \square)$ and $\operatorname{Vhom}(\square, N)$ are left exact functors. It is not true in general that $\operatorname{Vhom}(M, \square)$ and $\operatorname{Vhom}(\square, N)$ are (right) exact functors. Because, if we consider $V$ the vertex algebra given by an associative commutative algebra with unit and $d=0$, then everything collapses to the classical case, that is $\operatorname{Vhom}(M, N)=\operatorname{Vhom}^{r}(M, N)=\operatorname{Hom}_{V}(M, N)$ and we know that $\mathrm{Hom}_{V}$ is not exact in both variables. This is the motivation for the introduction of the notions of vertex projective and vertex injective $V$-modules.

Definition 7.2. A $V$-module $P$ is said to be vertex projective if for any surjective $V$-homomorphism $\psi: N \rightarrow N^{\prime \prime}$ and any $f \in \operatorname{Vhom}\left(P, N^{\prime \prime}\right)$, there exists a lifting $g$, that is, there exists $g \in \operatorname{Vhom}(P, N)$ making the following diagram commute:

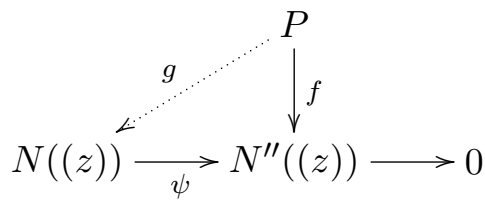

Proposition 7.3. A $V$-module $P$ is vertex projective if and only if $V h o m(P, \square)$ is an exact functor.

Proof. If $P$ is vertex projective and $\psi: N \rightarrow N^{\prime \prime}$ is a surjective $V$-homomorphism, then given $f \in \operatorname{Vhom}\left(P, N^{\prime \prime}\right)$ there exists a lifting $g: P \rightarrow N((z))$ with $f=\psi g=\psi_{*}(g) \in \operatorname{Im} \psi_{*}$, and so 
$\psi_{*}$ is surjective. Hence, by Theorem 7.1 we have that $\operatorname{Vhom}(P, \square)$ is an exact functor. The converse follows by reversing the previous argument.

Definition 7.4. A $V$-module $E$ is vertex injective if for any injective $V$-homomorphism $\varphi$ : $M^{\prime} \rightarrow M$ and any $f \in \operatorname{Vhom}\left(M^{\prime}, E\right)$, there exists $g \in \operatorname{Vhom}(M, E)$ making the following diagram commute:

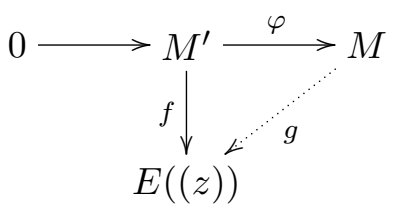

In words, every vertex homomorphism from a submodule into $E$ can always be extended to a vertex homomorphism from the big module into $E$.

Proposition 7.5. A $V$-module $E$ is vertex injective if and only if $V h o m(\square, E)$ is an exact functor.

Proof. By Theorem 7.1, $\operatorname{Vhom}(\square, E)$ is a left exact contravariant functor, therefore the thrust of the proposition is that the induced map $\varphi^{*}$ is surjective whenever $\varphi$ is injective, and this is exactly the definition of vertex injective module.

Now, we examine the behavior of tensor products with respect to exact sequences:

Theorem 7.6. (Right exactness) If $N^{\prime} \stackrel{f}{\longrightarrow} N \stackrel{g}{\longrightarrow} N^{\prime \prime} \longrightarrow 0$ is an exact sequence of $V$-modules and $M$ is a $V$-module, then

$$
M \underset{V}{\otimes} N^{\prime} \stackrel{1_{M} \otimes f}{\longrightarrow} M \underset{V}{\otimes} N \stackrel{1_{M} \otimes g}{\longrightarrow} M \underset{V}{\otimes} N^{\prime \prime} \longrightarrow 0
$$

is an exact sequence of $V$-modules.

Proof. There are three things to check.

(i) $1_{M} \otimes g$ is surjective: given $v^{\prime \prime} \in N^{\prime \prime}$ and $u \in M$, there exists $v \in N$ such that $g(v)=v^{\prime \prime}$, and hence

$$
u \underset{z}{\otimes} v^{\prime \prime}=u \underset{z}{\otimes} g(v)=\left(1_{M} \otimes g\right)(u \underset{z}{\otimes} v) .
$$

Since elements of type $u \underset{z}{\otimes} v^{\prime \prime}$ (in fact, the coefficients in $z$ ) generate $M \underset{V}{\otimes} N^{\prime \prime}$, we conclude that $1_{M} \otimes g$ is surjective.

(ii) $\operatorname{Im}\left(1_{M} \otimes f\right) \subseteq \operatorname{Ker}\left(1_{M} \otimes g\right)$ : it suffices to prove that the composite is 0 , but using Corollary 4.9 we have

$$
\left(1_{M} \otimes f\right)\left(1_{M} \otimes g\right)=1_{M} \otimes f g=1_{M} \otimes 0=0 .
$$

(iii) $\operatorname{Ker}\left(1_{M} \otimes g\right) \subseteq \operatorname{Im}\left(1_{M} \otimes f\right)$ : let $E=\operatorname{Im}\left(1_{M} \otimes f\right)$. By part (ii), $E \subseteq \operatorname{Ker}\left(1_{M} \otimes g\right)$, and so $1_{M} \otimes g$ induces a map $\hat{g}:(M \otimes N) / E \rightarrow M \otimes_{V} N^{\prime \prime}$ with $\hat{g}\left(u \otimes_{z} v+E\right)=u \otimes_{z} g(v)$, where $u \in M$ and $v \in N$. Now, if $\pi: M \otimes_{V} N \rightarrow\left(M \otimes_{V} N\right) / E$ is the canonical epimorphism, then $\hat{g} \pi=1_{M} \otimes g$. We shall define a map $h: M \otimes_{V} N^{\prime \prime} \rightarrow\left(M{ }_{V} N\right) / E$ such that $h \hat{g}=i d$. This obviously will imply that $\hat{g}$ is injective, and hence

$$
\operatorname{Ker}\left(1_{M} \otimes g\right)=\operatorname{Ker}(\hat{g} \pi)=\operatorname{Ker}(\pi)=\operatorname{Im}\left(1_{M} \otimes f\right)
$$


and we are done. First, we show that the map

$$
\tilde{h}_{z}: M \times N^{\prime \prime} \longrightarrow[(M \underset{V}{\otimes} N) / E]((z))
$$

given by $\tilde{h}_{z}\left(u, v^{\prime \prime}\right)=\pi\left(u \otimes_{z} v\right)$, where $g(v)=v^{\prime \prime}$, is well defined, i.e. is independent of the choice of $v$. Note that there is at least on such $v$ since $g$ is surjective. If $g\left(v_{1}\right)=g\left(v_{2}\right)=v^{\prime \prime}$, then $g\left(v_{1}-v_{2}\right)=0$, and by hypothesis, $v_{1}-v_{2}=f\left(v^{\prime}\right)$ for some $v^{\prime} \in N^{\prime}$. Then each coefficient in $z$ of

$$
u \otimes_{z} v_{1}-u \underset{z}{\otimes_{2}} v_{2}=u \otimes_{z}\left(v_{1}-v_{2}\right)=u \underset{z}{\otimes} f\left(v^{\prime}\right)
$$

is in $\operatorname{Im}\left(1_{M} \otimes f\right)=E$, and this proves that our map is well defined. It is obviously a vertex bilinear map, and by the universal property, there is a unique $V$-homomorphism

$$
h: M \underset{V}{\otimes} N^{\prime \prime} \longrightarrow(M \underset{V}{\otimes} N) / E
$$

such that $h\left(u \otimes_{z} v^{\prime \prime}\right)=\pi\left(u \otimes_{z} v\right)$, where $g(v)=v^{\prime \prime}$. It is clear that

$$
h\left(\hat{g}\left(u \otimes_{z} v+E\right)\right)=h\left(u \otimes_{z} g(v)\right)=u \otimes_{z} v+E,
$$

hence $h \hat{g}$ is the identity map. So, we conclude that $\hat{g}$ is injective, as was to be shown.

The next type of module arises from tensor products in the same way that vertex projective and vertex injective modules arise from $V h o m$. If $V$ is a vertex algebra given by an associative commutative algebra with unit, the tensor product coincides with the usual tensor product of modules and we know that the associated functor is not left exact.

Definition 7.7. A $V$-module $M$ is vertex flat if $M \underset{V}{\otimes} \square$ is an exact functor.

Because the functors $M \otimes \square$ are right exact, we see that a $V$-module $M$ is vertex flat if and only if, whenever $i \in \operatorname{Hom}_{V}^{V}\left(N^{\prime}, N\right)$ is an injection, then $1_{M} \otimes i: M \underset{V}{\otimes} N^{\prime} \rightarrow M \underset{V}{\otimes} N$ is also an injection.

Acknowledgements. The author was supported by a grant by Conicet, Consejo Nacional de Investigaciones Científicas y Técnicas (Argentina). The author would like to thank C. Boyallian, M. V. Postiguillo and C. Bortni for their constant help, care and support to finish this work. The author would like to thank F. Orosz for her help, patience, kindliness and constant support throughout the first part of this work.

\section{REFERENCES}

[BK] B. Bakalov and V. G. Kac, Field algebras, Internat. Math. Res. Notices 3 (2003), 123-159.

[Bo1] R. E. Borcherds, Vertex algebras, Kac-Moody algebras, and the Monster, Proc. Natl. Acad. Sci. USA, 83 (1986) 3068-3071.

[Bo2] R. E. Borcherds, Vertex algebras. Topological field theory, primitive forms and related topics (Kyoto, 1996), 35-77, Progr. Math., 160, Birkhuser Boston, Boston, MA, 1998.

[DLM] C. Dong, H. Li and G. Mason, Hom functor and the associativity of tensor products of modules for vertex operator algebras J. Algebra 188 (1997), no. 2, 443-475. MR1435368.

[FHL] I. B. Frenkel, Y.-Z. Huang and J. Lepowsky, On axiomatic approaches to vertex operator algebras and modules, Memoirs Amer. Math. Soc. 104, 1993.

[FZ] I. B. Frenkel and Y. Zhu, Vertex operator algebras associated to representations of affine and Virasoro algebras, Duke Math. J. 66 (1992), 123168.

[G] M. Gaberdiel, Fusion in conformal field theory as the tensor product of the symmetry algebra. Internat. J. Modern Phys. A 9 (1994), no. 26, 4619-4636.

$[\mathrm{H}] \quad$ Y.-Z. Huang, A theory of tensor products for module categories for a vertex operator algebra, IV, J. Pure Appl. Alg. 100 (1995) 173-216. 
[HL1] Y.-Z. Huang and J. Lepowsky, Toward a theory of tensor products for representations of a vertex operator algebra, in: Proc. 20th International Conference on Differential Geometric Methods in Theoretical Physics, New York, 1991 , ed. S. Catto and A. Rocha, World Scientific, Singapore, 1992, Vol. 1, 344- 354.

[HL2] Y.-Z. Huang and J. Lepowsky, Tensor products of modules for a vertex operator algebras and vertex tensor categories, in: Lie Theory and Geometry, in honor of Bertram Kostant, ed. R. Brylinski, J.-L. Brylinski, V. Guillemin, V. Kac, Birkhäuser, Boston, 1994, 349-383

[HL3] Y.-Z. Huang and J. Lepowsky, A theory of tensor products for module categories for a vertex operator algebra, I, Selecta Mathematica (New Series) 1 (1995), 699- 756.

[HL4] Y.-Z. Huang and J. Lepowsky, A theory of tensor products for module categories for a vertex operator algebra, II, Selecta Mathematica (New Series) 1 (1995), 757- 786.

[HL5] Y.-Z. Huang and J. Lepowsky, A theory of tensor products for module categories for a vertex operator algebra, III, J. Pure Appl. Alg. 100 (1995) 141-171.

[K] V. G. Kac, Vertex algebras for beginners, University Lecture Series, 10. American Mathematical Society, Providence, RI, 1996. Second edition 1998.

[KL1] D. Kazhdan and G. Lusztig, Affine Lie algebras and quantum groups, Internat. Math. Res. Notices 2 (1991), 21-29.

[KL2] D. Kazhdan and G. Lusztig, Tensor structures arising from affine Lie algebras, I, J. Amer. Math. Soc. 6 (1993), 905-947.

[KL3] D. Kazhdan and G. Lusztig, Tensor structures arising from affine Lie algebras, II, J. Amer. Math. Soc. 6 (1993), 949-1011.

[KL4] D. Kazhdan and G. Lusztig, Tensor structures arising from affine Lie algebras, III, J. Amer. Math. Soc. 7 (1994), 335-381.

[KL5] D. Kazhdan and G. Lusztig, Tensor structures arising from affine Lie algebras, IV, J. Amer. Math. Soc. 7 (1994), 383-453.

[LL] J. Lepowsky; H. Li, Introduction to vertex operator algebras and their representations. Progress in Mathematics, 227. Birkhuser Boston, Inc., Boston, MA, 2004. xiv+318 pp.

[Li1] H.-S. Li, Representation theory and tensor product theory for vertex operator algebras, Ph.D. thesis, Rutgers University, 1994 - arXiv: hep-th/9406211.

[Li2] H. Li, Local systems of vertex operators, vertex superalgebras and modules. J. Pure Applied Algebra 109 (1996), 143-195.

[Li3] H. Li, Axiomatic $G_{1}$-vertex algebras, Commun. Contemp. Math. 5 (2003), no. 2, 281-327.

[L1] J. Liberati, Cohomology of vertex algebras, arXiv:1603.07958 [math.QA].

[L2] J. Liberati, Tensor product of conformal modules over a Lie conformal algebra, to appear.

[L3] J. Liberati, Tensor product of modules over an H-pseudo algebra, to appear.

[MS] G. Moore and N. Seiberg, Classical and quantum conformal field theory, Comm. Math. Phys. 123 (1989), $177-254$

[O] F. Orosz, Algebra de Vértices, Undergraduate Thesis - Advisor: J. Liberati - Universidad Nacional de Córdoba, Facultad de Matemática, Astronomía y Física, 2011.

http://www.famaf.unc.edu.ar/institucional/biblioteca/trabajos/601/16294.pdf

[Zha] L. Zhang, Vertex tensor category structure on a category of Kazhdan-Lusztig, New York Jour. Math. 14 (2008), 261-284.

Ciem - CONICET, Medina Allende y Haya de la Torre, Ciudad Universitaria, (5000) Córdoba - Argentina. e-mail: joseliberati@gmail.com 